\title{
NLRP3 inflammasome pathway has a critical role in the host immunity against clinically relevant Acinetobacter baumannii pulmonary infection
}

\author{
N Dikshit ${ }^{1}$, SD Kale ${ }^{1}$, HJ Khameneh ${ }^{2}$, V Balamuralidhar ${ }^{1}, \mathrm{CY}$ Tang $^{3}$, P Kumar ${ }^{1}$, TP Lim ${ }^{4,5}$, TT Tan $^{6}$, \\ AL Kwa ${ }^{1,4,5,7}$, A Mortellaro ${ }^{2}$ and B Sukumaran ${ }^{1}$
}

The opportunistic Gram-negative bacterium Acinetobacter baumannii (AB) is a leading cause of life-threatening nosocomial pneumonia. Outbreaks of multidrug resistant (MDR)-AB belonging to international clones (ICs) I and II with limited treatment options are major global health threats. However, the pathogenesis mechanisms of various $A B$ clonal

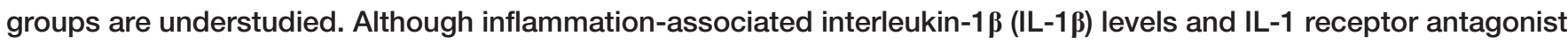
polymorphisms were previously implicated in MDR-AB-related pneumonia in patients, whether inflammasomes has any role in the host defense and/or pathogenesis of clinically relevant $A$. baumannii infection is unknown. Using a sublethal mouse pneumonia model, we demonstrate that an extensively drug-resistant clinical isolate (ICII) of $A$. baumannii exhibits reduced/delayed early pulmonary neutrophil recruitment, higher lung persistence, and, most importantly, elicits enhanced IL-1 $1 / / L-18$ production and lung damage through NLRP3 inflammasome, in comparison with $A$. baumanniitype strain. $A$. baumannii infection-induced IL-1ß/LL-18 production is entirely dependent on NLRP3-ASC-caspase-1/ caspase-11 pathway. Using Nlrp3 ${ }^{-\prime}$ mice infection models, we further show that while NLRP3 inflammasome pathway contributes to host defense against $A$. baumannii clinical isolate, it is dispensable for protection against $A$. baumanniitype strain. Our study reveals a novel differential role for NLRP3 inflammasome pathway in the immunity against clinically relevant $A$. baumannii infections, and highlights inflammasome pathway as a potential immunomodulatory target.

\section{INTRODUCTION}

Acinetobacter baumannii has recently emerged as one of the most successful Gram-negative bacteria causing both nosocomial and community-acquired infections globally. ${ }^{1,2}$ Although hospital-acquired pneumonia constitutes the most prevalent clinical manifestation associated with A. baumannii (AB), sepsis, urinary tract infection, meningitis, endocarditis, skin, and soft tissue infections are also common. ${ }^{3}$ Mortality rates of ventilatorassociated and community-acquired pneumonia caused by A. baumannii ranges from 40 to $70 \%{ }^{4,5}$ The clinical success of A. baumannii is attributed to its ability to (i) form biofilms and resist environmental stresses such as desiccation and disinfectants, (ii) develop anti-microbial resistance, (iii) acquire genetic material by horizontal gene transfer from unrelated genera, and (iv) adhere, colonize, and invade mammalian cells. ${ }^{6}$ In fact, because of its remarkable ability to develop resistance to nearly all known antibiotics, $A$. baumannii has recently been classified as a "red alert" human pathogen. ${ }^{7}$ This highlights the importance of developing new/improved or alternative strategies to combat A. baumannii.

Based on its virulence and ability to cause epidemics, A. baumannii is classified into three predominant clones (international clones (ICs) I, II, and III). Multidrug-resistant and carbapenem (the last resort of antibiotics to treat A. baumannii)-resistant virulent $A$. baumannii belonging to ICs I and II are the most prevalent clinically relevant

${ }^{1}$ Program in Emerging Infectious Diseases, Duke-NUS Medical School, Singapore, Singapore. ${ }^{2}$ Singapore Immunology Network (SlgN), Agency for Science, Technology and Research (A*STAR), Singapore, Singapore. ${ }^{3}$ Life Sciences Institute, National University of Singapore, Singapore, Singapore. ${ }^{4}$ Department of Pharmacy, Singapore General Hospital, Singapore. ${ }^{5}$ Sing Health Duke-NUS Medicine Academic Clinical Programme (MED ACP), Singapore, Singapore. ${ }^{6}$ Department of Infectious Diseases, Singapore General Hospital, Singapore, Singapore and ${ }^{7}$ Department of Pharmacy, National University of Singapore, Singapore, Singapore. Correspondence: B Sukumaran (bindu.sukumaran@duke-nus.edu.sg) 
A. baumannii strains causing outbreaks worldwide. ${ }^{1,8,9}$ ICII is the most successful clone of $A$. baumannii causing outbreaks in hospital environments worldwide, especially in Asia. However, what determines the clinical success of ICI and ICII clones is not well known. A thorough understanding of the host-pathogen interaction is critical for designing new therapeutics and vaccines. However, currently only limited information is available on the virulence mechanisms and associated protective host immune response, especially during virulent clinically relevant $A$. baumannii infections.

Development of pneumonia results from a complex interplay between the pathogen and the pulmonary mucosal immunity. Host innate immune system is critical for controlling A. baumannii infection, and may also have significant roles in immunopathogeneis associated with A. baumannii infection. The acute rapid infection kinetics of $A$. baumannii infections also further highlights the importance of innate immunity in controlling A. baumannii. Individuals with suppressed immune status are particularly vulnerable to A. baumannii infections. ${ }^{10}$ Immunomodulatory therapies in patients with compromised immune system could offer an attractive strategy to tackle A. baumannii infections. However, a thorough understanding of host immune response to A. baumannii infection is required to tackle effectively the infectious threat of $A$. baumannii infection. Previous studies have identified some of the innate immune mechanisms involved in host response to A. baumannii infection. Neutrophils and Toll-like receptor 4 (TLR4) pathway have important roles in the pulmonary innate immune defense against A. baumannii infection. ${ }^{11-15}$ However, TLR2 pathway has either a protective or pathological role during pulmonary A. baumannii infection. ${ }^{16}$ The role of intracellular innate immune receptors in A. baumannii immunity is only beginning to be appreciated. A role for intracellular NOD1/2 pathway in the innate immune response to $A$. baumannii infection was identified previously. ${ }^{17}$ A recent report has also demonstrated the role of the intracellular receptor TLR9 in the pulmonary host defense against A. baumannii. ${ }^{18}$ All these previous studies used either sensitive strains (not clinically relevant drug-resistant forms) of A. baumannii or used mouse sepsis models of infection and hence may not accurately depict the interaction of clinically relevant $A$. baumannii with the pulmonary innate immune system.

Using mouse pneumonia infection models, previous studies have shown that clinically relevant more virulent forms of A. baumannii (belonging to ICI and II) survive and persist in the lungs of the host by inducing only a weak innate immune inflammatory response in comparison with less/nonvirulent forms. ${ }^{19,20}$ This implies that drug-resistant virulent $A$. baumannii may evade/suppress the innate immune response to persist and cause various pathological consequences in the host. However, the underlying mechanism or the innate immune pathways involved in this are not fully characterized yet.

Inflammasomes are important intracellular multiprotein innate immune complexes that are implicated in infection control, inflammation, and tissue injury. An earlier study conducted in patients with $A$. baumannii-associated pneumonia has showed that higher concentration of interleukin-1 $\beta$ (IL-1 $\beta$ ) (a marker of inflammasome activation) observed in the bronchoalveolar lavage (BAL) of patients correlated with the higher bacterial burden or the presence of virulent bacteria in their alveoli. ${ }^{21}$ This study has proposed that levels of BAL IL-1 $\beta$ could be considered as a marker for ongoing inflammation in response to persistent bacterial infection in the lung. Yet, another clinical study showed that a polymorphism in the gene encoding the anti-inflammatory antagonist of IL-1 receptor (IL-1RA) (a competitive inhibitor of IL-1 $\beta$ ) is associated with the risk of developing multidrug-resistant $A$. baumannii-related pneumonia. ${ }^{22}$ These studies clearly indicated an involvement of IL-1associated pathways in the pulmonary immune response to clinically relevant $A$. baumannii infection. However, the role of inflammasome in the host immune response to clinically important $A$. baumannii is not characterized.

Inflammasomes sense either the microbial stimuli or danger signals and regulates two major host protective responses: (a) secretion of highly proinflammatory cytokines IL- $1 \beta$ and IL-18 and (b) induction of pyroptosis. ${ }^{23}$ The best-characterized inflammasome is the NLRP3 inflammasome. NLRP3 has been implicated in various pulmonary diseases including acute respiratory distress syndrome, sepsis, and several pulmonary bacterial and viral infections including Klebsiella pneumoniae, Streptococcus pneumoniae, Staphylococcus aureus, Chlamydia pneumoniae, Mycobacterium tuberculosis, Legionella pneumophila, influenza virus, human rhinovirus, respiratory syncytial virus, and Aspergillus fumigatus (for a review see Chaput et $\left.a .^{24}\right)$. In addition, differential activation of NLRP3 inflammasome by bacterial pathogens of varying virulence resulted in different pathological outcomes. ${ }^{25}$ However, (i) whether NLRP3 inflammasome pathway has any role in the host defense to A. baumannii infection and (ii) whether there is any differential interaction of virulent $A$. baumannii with NLRP3 that can impact the clinical outcomes associated with A. baumannii infections is also currently not known.

In this report, we investigated the hypothesis that clinically relevant antibiotic-resistant forms of A. baumannii belonging to ICI and ICII may interact differently with the inflammasome pathway leading to host defense and also pathological outcomes. Particularly, we aimed to understand the interaction of A. baumannii with the pulmonary NLRP3 inflammasome system. For this study, we used an extensively drug-resistant (XDR) A. baumannii isolate (AB-8879, belonging to ICII clonal group) that caused severe sepsis and bacteremia in patients of the burns intensive care unit. ${ }^{26,27} \mathrm{AB}-8879$ strain belongs to carbapenemresistant $A$. baumannii, and in addition, it shows polymyxin $\mathrm{B}$ heteroresistance. The results of our study revealed that the clinical A. baumannii isolate caused a predominant activation of NLRP3 inflammasome in vivo, and that NLRP3 was important during host immune response to A. baumannii infection.

\section{RESULTS}

A. baumannii infection primes and activates the inflammasome complex in mouse bone marrow-derived macrophages

IL-1 $\beta$ levels/IL-1RA polymorphism are previously reported to be associated with multidrug-resistant- $\mathrm{AB}$-related pneumonia 
in patients ${ }^{21,22}$, indicating that inflammasome activation could have a role in clinically relevant $A$. baumannii infections. Hence, we aimed to investigate the role of inflammasomes during clinically relevant $A$. baumannii (belonging to ICI and II implicated in outbreaks worldwide) infections. An XDR clinical isolate of A. baumannii causing severe sepsis and bacteremia in patients in burns intensive care unit from Singapore belonging to the ICII (carbapenem-resistant (8879, ICII) $)^{26,27}$ was used for our studies. We first ascertained whether A. baumannii can activate the inflammasome pathway in macrophages. Bone marrow-derived macrophages (BMDMs) were infected with AB-8879. Transcript levels of procaspase- 1 and pro-IL-1 $\beta$ were determined by quantitative reverse transcription-PCR. A. baumannii infection led to 5.5 -fold increase in procaspase- 1 mRNA $(P=0.004)$ (Figure 1a) and 344-fold increase in pro-IL-1 $\beta$ mRNA $(P=0.003)$ levels (Figure $1 \mathbf{b})$ at $24 \mathrm{~h}$ after infection. Next, to functionally verify whether inflammasome complex is also activated at the protein level, we first determined the levels of activated caspase- 1 by western blot analysis. A. baumannii infection resulted in the cleavage of procaspase-1 to its active form caspase-1 p20 (Figure 1c). We next examined whether the caspase- 1 activation observed during A. baumannii infection further leads to the generation of IL- $1 \beta$ and IL-18, by measuring these cytokines in the infected BMDM culture supernatants. The release of IL-1 $\beta$ (Figure 1d) and IL-18 (Figure 1e) was found to be induced upon A. baumannii infection of lipopolysaccharide (LPS)-primed BMDMs. LPS + ATP-treated cells were used as positive controls. A. baumannii was also able to prime the inflammasome as it mediated IL-1 $\beta$ (Figure 1f) and IL-18 (Figure 1g) release in the absence of external LPS prestimulation. These results clearly demonstrate that A. baumannii is capable of priming as well as activating the inflammasome complex.

\section{A. baumannii induces IL-1 $\beta$ and IL-18 production through NLRP3 inflammasome}

NLRP3 and NLRC4 are major inflammasome complexes that can independently sense bacterial pathogens and lead to the production of IL-1 $\beta$ and IL-18 cytokines. NLRC 4 complex is activated by bacterial flagellin; however, the bacterial ligands for NLRP3 inflammasome are still unknown. As A. baumannii is a non-flagellated bacterium, we hypothesized that the infectioninduced IL-1 $\beta /$ IL-18 production would be likely through the activation of NLRP3 pathway. We first determined the expression of NLRP3 at the mRNA level and found that it was upregulated by 3.5 -fold $(P=0.006)$ upon $A$. baumannii infection (Figure 2a). Next, we infected BMDMs from wildtype C57BL/6 mice (WT) or Nlrp3-knockout (Nlrp3 ${ }^{-1-}$ ) mice and measured the production of catalytically active caspase-1 by western blot. While the BMDMs from WT mice produced mature (active) caspase-1, Nlrp3 ${ }^{-1-}$ macrophages failed to generate active caspase-1 following A. baumannii infection (Figure 2b). As caspase-1 processing is fully dependent on Nlrp3, a putative role of Nlrc4 in A. baumannii infection was excluded. In accordance with the lack of caspase- 1 activation,
IL-1 $\beta$ and IL-18 (Figure 2c,d) production was also completely abrogated in Nlrp3 ${ }^{-1-}$ macrophages upon A. baumannii infection. The production of MIP-2 (Figure 2e) and tumor necrosis factor- $\alpha$ (Figure 2f) were unaffected by the absence of NLRP3, indicating that the absence of NLRP3 does not lead to a generalized defect in inflammatory cytokine production and that NLRP3 is specifically involved in the synthesis of inflammatory IL-1 $\beta$ and IL-18 cytokines. To rule out the possibility that the reduced levels of IL-1 $\beta /$ IL-18 in Nlrp3 ${ }^{-1-}$ macrophages is due to reduced intracellular invasion of bacteria, the intracellular bacterial load in BMDM was ascertained at $4 \mathrm{~h}$ after infection. As seen in Supplementary Figure S1a online, there was no significant difference in the intracellular bacterial load between WT and Nlrp3 ${ }^{-1-}$ macrophages.

NLRP3 inflammasome is activated by various DAMPs such as ROS, cathespin B, and potassium ion efflux. ${ }^{28}$ We wanted to explore which of these mediators (if any) are involved in activating the inflammasome pathway during A. baumannii infection. We first explored if cellular invasion of bacteria is required to generate IL-1 $\beta$ through the above-mentioned mechanisms. For this, BMDMs were treated with cytochalasin $D$, which inhibits actin polymerization and therefore phagocytosis of bacteria. IL-1 $\beta$ release was found to be inhibited by 1.6 -fold by cytochalasin $\mathrm{D}$ treatment (Figure 2g). Heat-killed bacteria also elicited 1.7-fold lesser IL-1 $\beta$. As ROS has been documented in the control of A. baumannii, ${ }^{14,29}$ we next treated the cells with ROS inhibitor APDC to see whether ROS are involved in the generation of IL-1 $\beta$. We found that APDC treatment inhibited A. baumannii-induced IL-1 $\beta$ release by 1.7 -folds (Figure $2 \mathrm{~g}$ ). Treatment with $\mathrm{KCl}$, a potassium efflux inhibitor, reduced the IL-1 $\beta$ release by 1.6 -folds (Figure $\mathbf{2 g}$ ). Cathepsin B is a potential NLRP3 activator, which is released upon lysosomal damage. Pharmacological inhibition of cathepsin B by Ca-074-Me also inhibited IL-1 $\beta$ release by 1.5 -fold (Figure $2 \mathrm{~g}$ ). Since blocking none of the tested DAMPs fully abrogated IL-1 $\beta$ release, we conclude that each of the DAMPs evaluated may contribute only partially to $A$. baumannii-induced inflammasome activation. Therefore, these results show that upon A. baumannii infection, macrophages release various DAMPs, which may contribute to NLRP3 inflammasome activation.

Having found the role of NLRP3 pathway during A. baumannii infection, we next characterized the downstream signaling components of NLRP3 pathway that is involved in the caspase-1 activation/IL-1 $\beta$ generation during $A$. baumannii infection. As ASC is the adaptor protein and a major downstream component of NLRP3 inflammasome, we further assessed whether ASC is also involved in caspase-1 activation during A. baumannii infection. Infected $\mathrm{Asc}^{-1-}$ macrophages failed to generate active caspase- 1 p20, implying that ASC is required for caspase-1 activation during $A$. baumannii infection (Figure 3a). NLRP3/ASC complex ultimately recruits Caspase- 1 to the inflammasome. Caspase-1 cleaves pro-IL-1 $\beta$ and pro-IL-18 to their active forms either 


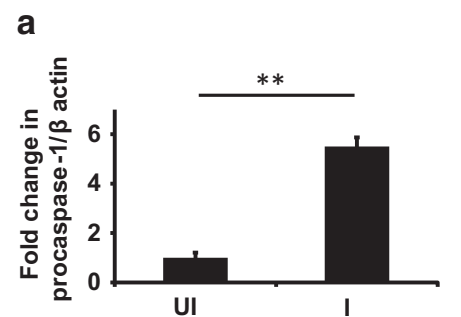

b

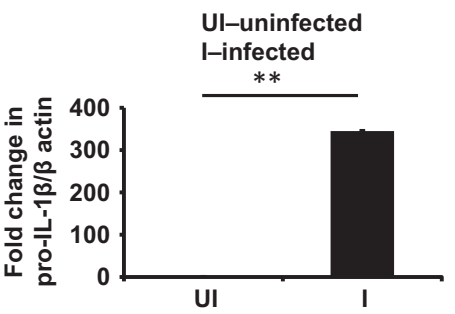

C

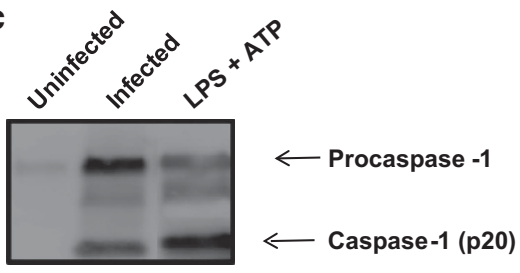

d

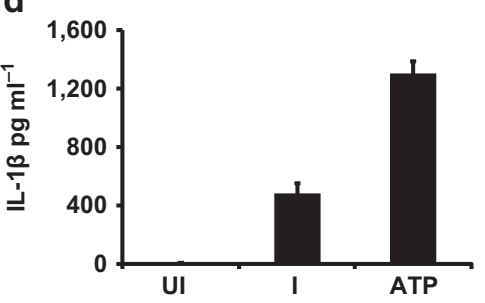

e Ul-uninfected
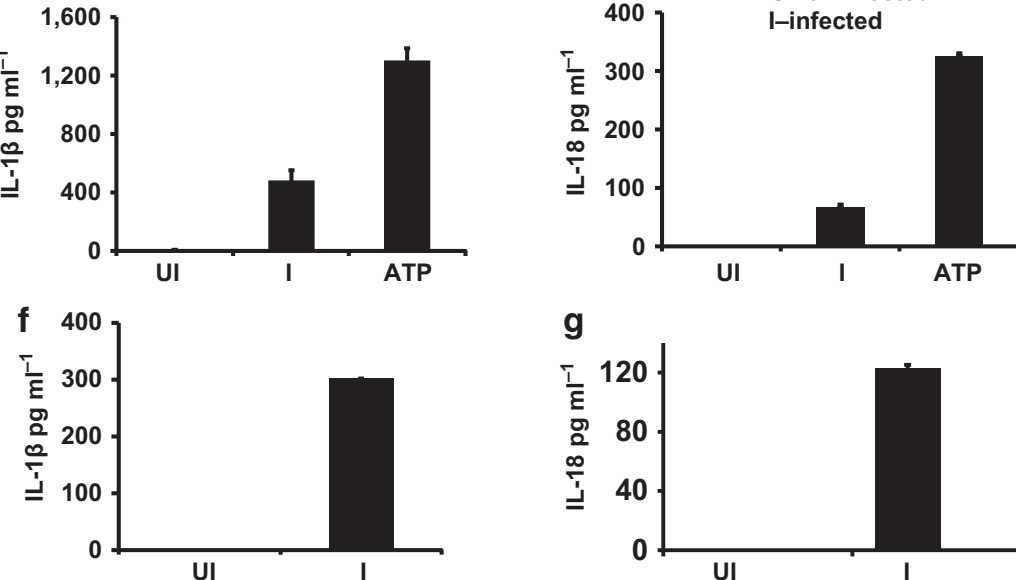

g

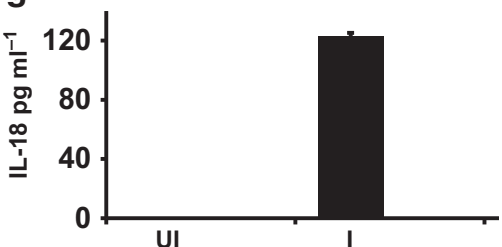

Figure 1 A. baumannii infection primes and activates the inflammasome complex in mouse bone marrow-derived macrophages (BMDMs). (a and $\mathbf{b}$ ) BMDMs were infected with $A$. baumannii-8879 (AB-8879) at a multiplicity of infection (MOI) of 100. At $24 \mathrm{~h}$ after infection, RNA was isolated and the mRNA expression of various genes was analyzed by reverse transcription-polymerase chain reaction (RT-PCR). mRNA levels of (a) procaspase-1 and (b) pro$\mathrm{IL}-1 \beta$ were upregulated with infection. (c-e) BMDMs were stimulated with $100 \mathrm{ng} \mathrm{ml}^{-1}$ lipopolysaccharide (LPS) overnight before $A$. baumannii infection at an $\mathrm{MOI}$ of 100 . The culture supernatants were collected at $4 \mathrm{~h}$ after infection and analyzed for (c) caspase-1 p20 by western blot and for cytokines (d) IL-1 $\beta$ and (e) IL-18 by enzyme-linked immunosorbent assay (ELISA). LPS + ATP-treated cells were used as a positive control for cytokine production. ( $f$ and $\mathbf{g}$ ) Unprimed BMDMs were infected with AB-8879 at an MOI of 100 . The culture supernatants were collected at $24 \mathrm{~h}$ after infection and analyzed for cytokines (f) IL-1 $\beta$ and $(\mathbf{g})$ IL-18 by ELISA. ${ }^{*} P<0.01$. Experiments $(\mathbf{a}, \mathbf{b}, \mathbf{d}, \mathbf{e}, \mathbf{f}$ and $\mathbf{g})$ were performed three times with similar results and data represent mean \pm s.d. of technical triplicates of a representative experiment. Experiment (c) was repeated two times with similar results. Representative blot is shown.

independently or under the regulation of Caspase- $11 .{ }^{30}$ A. baumannii failed to activate Caspase- 1 in Casp-11 $-1-$ (single knockout) macrophages (Figure 3a). Casp-1 ${ }^{-/-}$ macrophages were used as positive controls. Macrophages from Asc ${ }^{-I-}$, Casp-1 ${ }^{-1-}$, and Casp-11 ${ }^{-/-}$single-knockout or Casp-1/11 ${ }^{-1-}$ double-knockout mice failed to generate IL-1 $\beta$ (Figure 3b) or IL-18 (Figure 3c) cytokines upon A. baumannii infection. However, the induction of transcripts of IL- $1 \beta$ and IL-18 was not affected by the absence of Nlrp3, Asc, Caspase-1, or Caspase-11 (Supplementary Figure S1b,c, respectively). Thus, we conclusively prove that the production of IL-1 $\beta$ and IL-18 during A. baumannii infection is driven by both canonical and noncanonical NLRP3 inflammasome pathway, and also suggests that NLRP3 inflammasome pathway may have a role in the host immunity to A. baumannii infections.
Clinical isolate of $\boldsymbol{A}$. baumannii elicits enhanced NLRP3-dependent production of IL-1 $\beta$ and IL-18 and pyroptosis in the lungs in comparison with the type strain in vivo

We next assessed the in vivo role of NLRP3 inflammasome during A. baumannii pneumonia by the clinical isolate and also examined if inflammasome activation is a common feature of all A. baumannii strains. We used AB-8879 isolate (as a representative of clinically relevant $A$. baumannii), and for the comparison purpose, we used the type strain 19606 (an antibiotic-susceptible strain that has been used extensively for pathogenesis studies before) and compared their ability to activate inflammasome pathway using a mouse pneumonia model. As our aim was to study host immune response to A. baumannii pneumonia infection, we used a sublethal (selflimiting) pneumonia model using immunocompetent mice. 


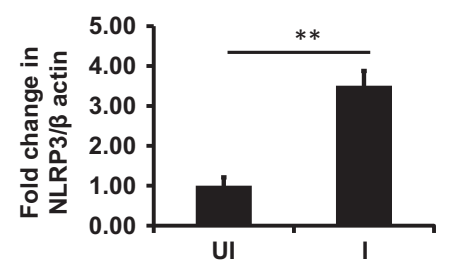

C

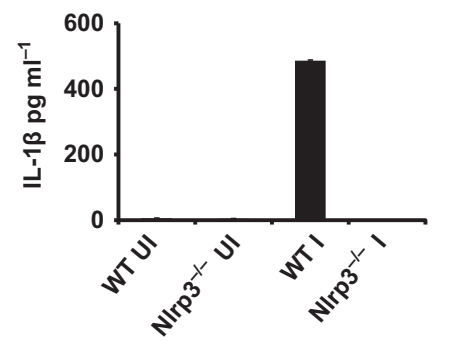

e

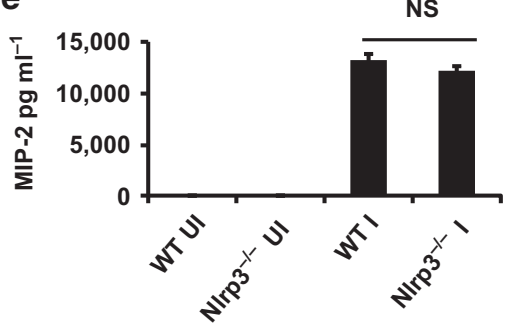

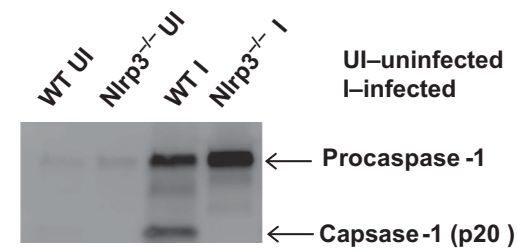

d
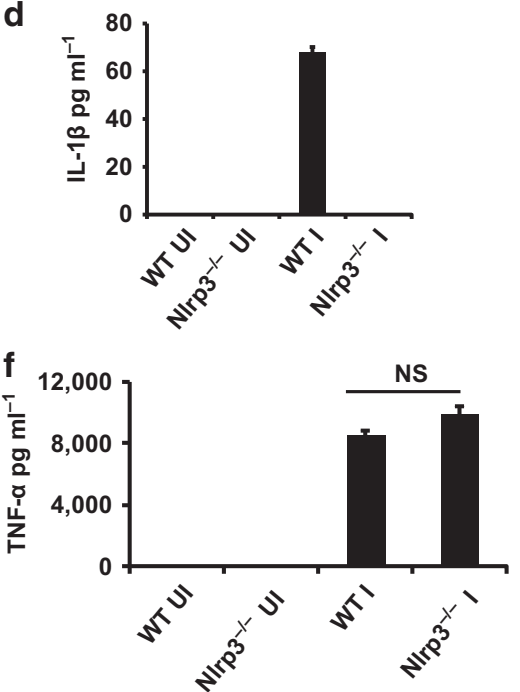

g

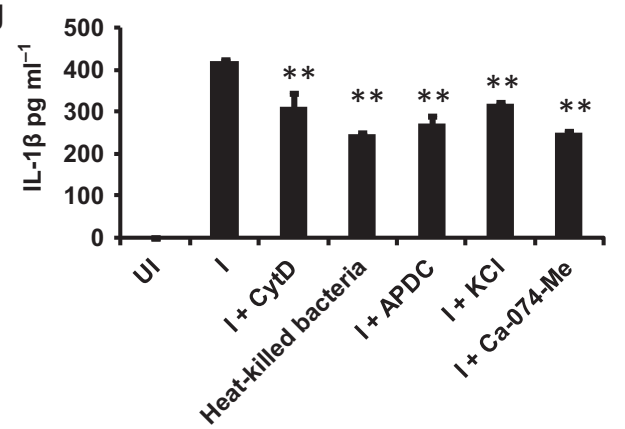

Figure 2 A. baumanniiinduces IL-1 $\beta$ and IL-18 production through NLRP3 inflammasome. (a) Bone marrow-derived macrophages (BMDMs) from wildtype C57BL/6 (WT) mice were infected with A. baumannii 8879 (AB-8879) at a multiplicity of infection (MOI) of 100. At $24 \mathrm{~h}$ after infection RNA was isolated and the mRNA expression of Nlrp3 was analyzed by quantitative reverse transcription-PCR (qRT-PCR). (b-f) BMDMs from wild-type C57BL/6 mice (WT) or Nlrp3-knockout mice (Nlrp3 ${ }^{-1}$ ) were infected with AB-8879 at an MOI of 100 . The culture supernatants were collected at $24 \mathrm{~h}$ after infection and analyzed for (b) caspase-1 p20 by western blot or for (c) IL-1 $\beta$, (d) IL-18, (e) MIP2, and (f) tumor necrosis factor- $\alpha$ (TNF- $\alpha$ ) by enzyme-linked immunosorbent assay (ELISA). (g) BMDMs from wild-type C57BL/6 mice were treated with $\mathrm{KCl}$ (prevents potassium ion efflux), cytochalasin D (Cyt D) (actin reorganization inhibitor), APDC (ROS inhibitor), or CA-074-Me (cathepsin B inhibitor) $1 \mathrm{~h}$ before infection with AB-8879 and subsequently throughout the course of infection. BMDMs were also infected with heat-killed $A$. baumannii. At $24 \mathrm{~h}$ after infection, culture supernatants were collected and analyzed for IL-1 $\beta$ by ELISA. ${ }^{* *} P<0.01$. Statistical analysis were conducted using using $t$-test except in $\mathbf{g}$, where analysis was conducted using oneway analysis of variance (ANOVA) $(F(4,5)=70.75, P=0.0001)$. Experiments were performed three times with similar results and data represent mean \pm s.d. of results of (a) three independent experiments or the $(\mathbf{c}-\mathbf{g})$ technical triplicates of a representative experiment. Experiment (b) was repeated two times with similar results. Representative blot is shown. NS, not significant.

Based on previous published reports from other research groups $^{5,15}$ and our unpublished data, a sublethal $\left(5 \times 10^{7} \mathrm{CFU}\right)$ mouse pneumonia model for $A$. baumannii infection in C57BL/6 mice was used for the studies.

C57BL/6 mice were intranasally infected with $5 \times 10^{7} \mathrm{CFU}$ of A. baumannii using either AB-8879 or AB-19606, and the levels of inflammasome-associated cytokines IL- $1 \beta$ and IL-18 was assessed in the BAL fluid at 4 and $24 \mathrm{~h}$ after infection. Only low levels of IL- $1 \beta$ were detected in the BAL of either
AB-8879- or AB-19606-infected mice at $4 \mathrm{~h}$ after infection (Figure 4a). Moreover, the levels of IL-1 $\beta$ were comparable between the strains at this time point. Similarly, although both AB-8879 and AB-19606 produced measurable amounts of IL-18 at $4 \mathrm{~h}$ after infection, the levels were comparable between the strains (Figure $\mathbf{4 b}$ ). However at $24 \mathrm{~h}$ after infection, AB-8879 elicited robust production of both IL- $1 \beta$ and IL-18 cytokines (Figure 4a,b). However, interestingly, these cytokines were produced only in significantly lesser 
a

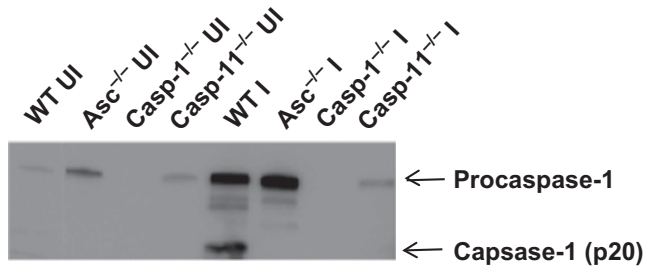

b
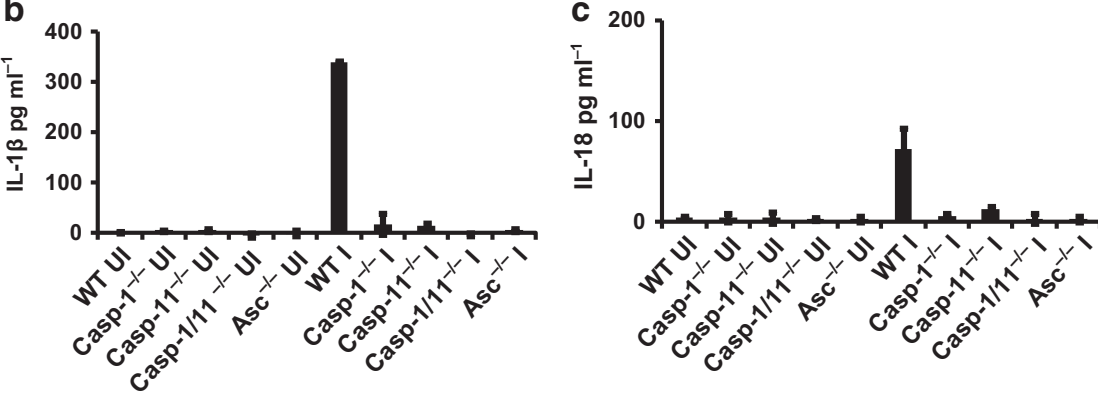

Figure $3 \mathrm{IL}-1 \beta$ and IL-18 production during $A$. baumannii infection is driven by ASC/Caspase-11/Caspase-1 pathway. (a) Bone marrow-derived

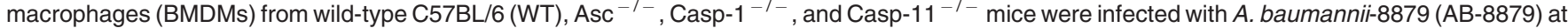
a multiplicity of infection (MOI) of 100 . At $24 \mathrm{~h}$ after infection, the culture supernatant was analyzed for caspase-1 p20 by western blot. (b and $\mathbf{c}$ ) BMDMs

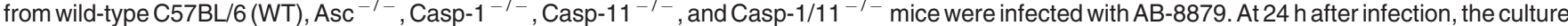
supernatant was collected and analyzed for (b) IL-1 $\beta$ and (c) IL-18 by enzyme-linked immunosorbent assay (ELISA). Experiments (b and c) were performed three times with similar results and data represent mean \pm s.d. of the technical triplicates of a representative experiment. Experiment (a) was performed two times with similar results. Representative blot is shown.

amounts by $\mathrm{AB}-19606$ strain as compared with $\mathrm{AB}-8879$ (IL-1 $\beta$ was 3.5 -fold lesser, $P=0.03$, IL- 18 was 3 -fold lesser, $P=0.04$ ) (Figure 4a,b). Intriguingly, both AB-8879 and AB-19606 elicited comparable levels of both IL-1 $\beta$ and IL-18 during ex vivo stimulation of mouse BMDMs (Supplementary Figure S2a,b, respectively). Moreover, a dose-response analysis also showed that comparable levels of IL- $1 \beta$ was produced ex vivo by AB-19606 and AB-8879 at all the multiplicity of infections (MOIs) and at both time points tested (4 and $24 \mathrm{~h}$ after infection) (Supplementary Figure S2c,d, respectively). These experiments indicate that AB-8879 may differentially interact with the host immune system in vivo, thereby leading to the enhanced inflammasome activation. Additionally, AB-8879 induced higher cell death/lung damage as evidenced by higher lactate dehydrogenase $(\mathrm{LDH})$ levels $^{31}$ in the BAL from AB-8879-infected mice in comparison with AB-19606-infected mice (1.5-fold difference, $P=0.04$ ) (Figure $4 c$ ). Cell death can also be mediated by caspase-11-mediated pathway that is associated with the secretion of IL- $1 \alpha$, a related cytokine to IL- $1 \beta .^{32}$ Notably, we also observed higher levels of IL- $1 \alpha$ in the $\mathrm{BAL}$ of $\mathrm{AB}-8879$-infected mice in comparison with mice infected with AB-19606 (Supplementary Figure S3). These results clearly demonstrate that $\mathrm{AB}-8879$ induces robust inflammasome activation and cell death in vivo. Similar to our in vitro results, our in vivo infection experiments using Nlrp3 $3^{-1-}$ mice showed that the IL- $1 \beta$ and IL- 18 production by AB-8879 was completely dependent on NLRP3 inflammasome (Figure 4d,e). Additionally, cell death/lung damage was significantly reduced in Nlrp3 $3^{-1-}$ mice (1.5-fold lesser LDH $(P=0.04)$ was detected in BAL of Nlrp3 $3^{-1-}$ mice; Figure 4f). Thus, NLRP3 inflammasome pathway contributes to IL-1 $\beta /$ IL-18 production, and cell death/lung damage associated with clinically relevant AB-8879 infection.

We next explored the mechanism behind the enhanced ability of AB-8879 to activate inflammasome pathway in vivo. We observed that although there was no significant difference in the bacterial load between the AB-19606 and AB-8879 at early time points $(4 \mathrm{~h})$, the mice infected with the $A B-8879$ had significantly higher bacterial load in the lungs at all the late time points tested $(24,48$, and $72 \mathrm{~h}$ after infection) (Figure 5a). AB-8879 persisted in the lungs even at $72 \mathrm{~h}$ after infection; however, all the mice cleared the infection by $96 \mathrm{~h}$. In addition, AB-8879 successfully disseminated to extrapulmonary organs (such as the blood and spleen) at all the tested time points (Figure $\mathbf{5 b}, \mathbf{c}$, respectively). In contrast, we found that as per previous published reports, the type strain AB-19606 was completely cleared from the lungs by $\sim 48 \mathrm{~h}$ after infection, and there was minimal or no extra pulmonary dissemination (Figure 5a). Consistent with the higher bacterial load and delayed bacterial clearance, we also observed significant weight loss in the mice infected with the AB-8879 strain. The higher lung bacterial burden observed in mice infected with $\mathrm{AB}-8879$ could be one of the potential factors contributing to the enhanced activation of NLRP3 inflammasome pathway by AB-8879 in comparison to AB-19606.

It is interesting to note that there was no significant difference in the growth rate of $\mathrm{AB}-8879$ in comparison with AB-19606 in vitro. ${ }^{33}$ This ruled out the possibility that higher intrinsic growth rate of AB-8879 accounts for its increased load in vivo compared to AB-19606, but rather points towards the possibility of an immune evasion mechanism by the clinical isolate. 
a

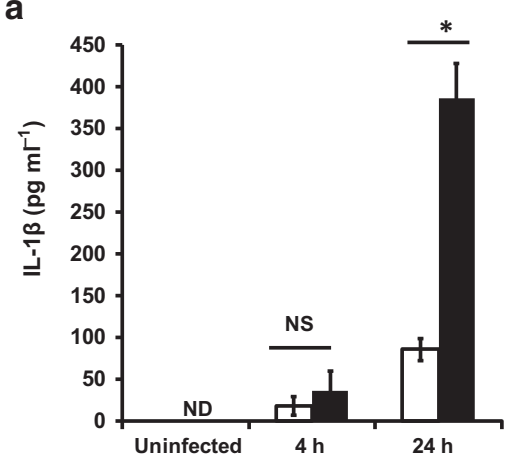

C
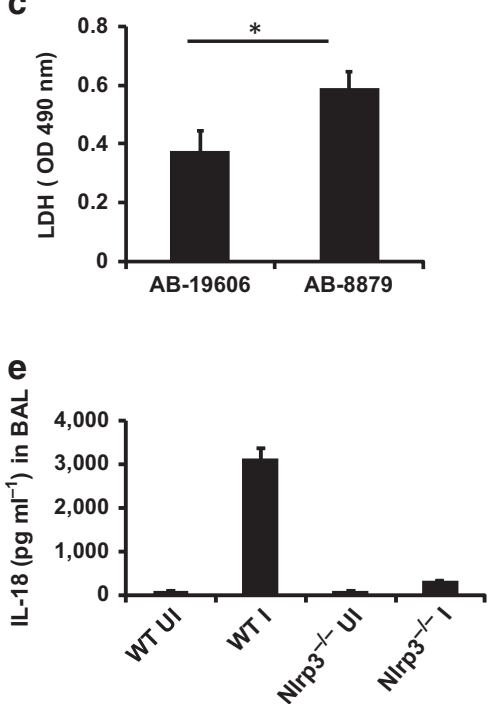

b

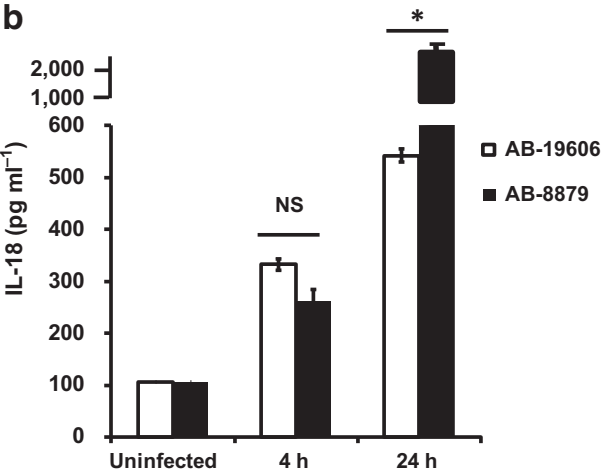

d

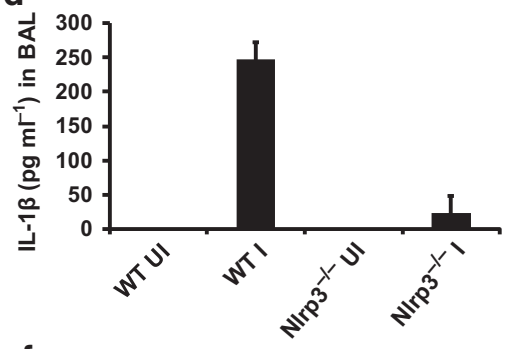

f

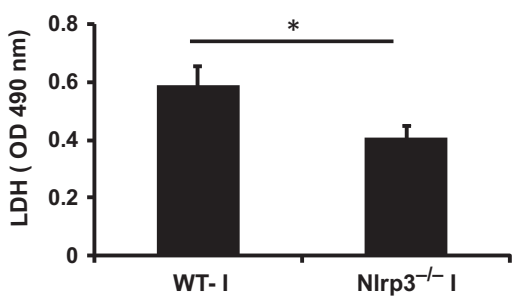

Figure 4 Clinical isolate of $A$. baumannii elicits enhanced NLRP3-dependent production of IL-1 $\beta$ and IL-18 and pyroptosis in the lungs in comparison with the type strain in vivo. (a-c) Wild-type C57BL/6 mice were infected with $5 \times 10^{7}$ colony-forming unit (CFU) of $A$. baumannii 19606 (AB-19606) or 8879 (AB-8879). At (a and b) $4 \mathrm{~h}$ and (a-c) $24 \mathrm{~h}$ after infection, mice were euthanized and bronchoalveolar lavage (BAL) was obtained and the levels of (a) IL-1 $\beta$, (b) IL-18, and (c) lactate dehydrogenase (LDH) were analyzed. (d-f) Wild-type C57BL/6 (WT) or NIrp3-knockout (NIrp3 ${ }^{-1-}$ ) mice were infected with $A$. baumannii 8879 (AB-8879). At $24 \mathrm{~h}$ after infection, mice were euthanized and BAL was obtained and the levels of (d) IL-1 $\beta$, (e) IL-18, and (f) LDH were analyzed as described in the Methods section. ${ }^{*} P \leq 0.05$. Experiments were performed three times with similar results and data represent mean \pm s.d. of the technical triplicates of a representative experiment. I, infected; UI, uninfected.

\section{A. baumannii clinical isolate exhibits reduced early neutrophil influx in the lungs in comparison to the type strain}

Since clinical isolate of A. baumannii attained higher bacterial load compared with type strain in vivo, we hypothesized that virulent $A$. baumannii potentially delays/evades/inhibits some of the early innate immune responses, leading to increased bacterial load during the early course of infection, and thereby resulting in enhanced activation of inflammasome pathway. We further reasoned that AB-19606 is effectively controlled by innate immune control mechanisms in the early stages of infection itself, resulting in diminished inflammasome activation. Early recruitment of neutrophils has a critical role in host resistance to respiratory $A$. baumannii infection. ${ }^{11,13-15}$ Therefore, we first sought to determine if there is any difference in the lung/BAL neutrophil recruitment kinetics of $\mathrm{AB}-8879$ and AB-19606. Neutrophil numbers were determined by immunostaining for myeloperoxidase (MPO). We found that the mice infected with AB-8879 had 2.5-fold lesser MPO-positive cells in the lungs as compared with AB-19606-infected mice at $4 \mathrm{~h}$ after infection $(P=0.03)$ (Figure 6a and Supplementary Figure S4a). Similarly, 2.3-fold lesser MPO-positive cells was observed in the BAL of mice infected with AB-8879 as compared with AB-19606 at $4 \mathrm{~h}$ after infection $(P=0.04)$ (Figure 6b). However, no difference in MPO-positive cells in lungs/BAL was observed among mice infected with either of the two strains at $24 \mathrm{~h}$ after infection (Figure 6a and Supplementary Figure S4b for the lungs and Figure $\mathbf{6 b}$ for the BAL, respectively). Further experiments were carried out to confirm the differences in the neutrophil numbers observed at $4 \mathrm{~h}$ after infection. MPO levels in lungs were also determined by enzyme-linked immunosorbent assay (ELISA) and were found to be 1.3 -fold lesser in mice infected with $\mathrm{AB}-8879$ as compared with mice infected with AB-19606 $(P=0.01)$ (Figure 6c). Neutrophil elastase activity was found to be 2.4 -fold lesser in BAL of mice infected with AB- 8879 than in the BAL from mice 

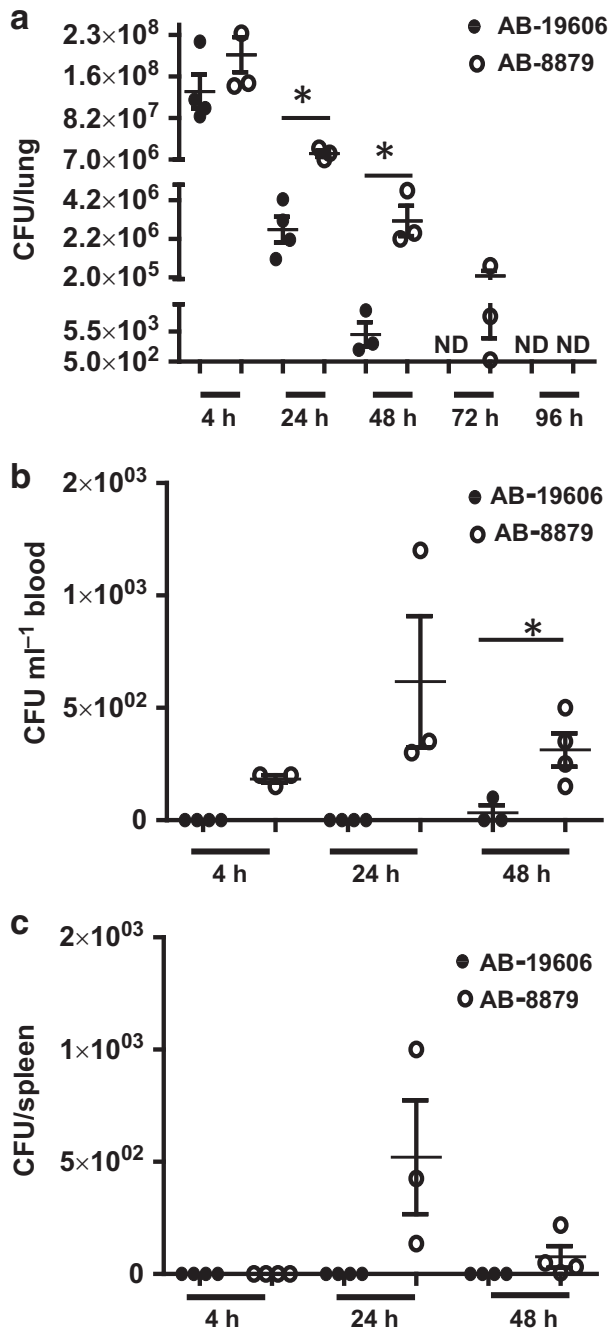

Figure 5 A. baumannii clinical isolate exhibits higher bacterial load and extrapulmonary dissemination in comparison with the type strain. Wild-type C57BL/6 mice were infected with $5 \times 10^{7}$ colony-forming unit (CFU) of A.baumannii 19606 (AB-19606) or 8879 (AB-8879) ( $n=3-5$ mice per group). At the indicated time points after infection, mice were euthanized. Bacterial load was determined in the (a) lungs and (c) spleen by homogenizing the organs in $0.1 \%$ Triton $\mathrm{X}-100$ and plating on trypticase soy agar (TSA) plates as described in the Methods section. Bacterial load in the (b) blood were enumerated by directly plating blood on TSA plates as described in the Methods section. ${ }^{\star} P \leq 0.05$. Data are represented as mean \pm s.d. Data are representative of three independent experiments with similar results. ND, not detectable.

infected with AB-19606 ( $P=0.02)$ (Figure 6d). Furthermore, Giemsa staining of the BAL at $4 \mathrm{~h}$ after infection also showed that AB-8879-infected mice had twofold lesser number of neutrophils as compared with AB-19606 $(P=0.005)$ (Figure 6e). Consistent with this, there was significantly lesser number of total immune cells in the BAL of mice infected with AB-8879 in comparison with that from AB-19606 (Figure 6e). However, the percentage of alveolar macrophages was comparable in AB-19606 and AB-8879 at this time point (Figure 6e). The percentage of other immune cells was negligible. We also performed FACS analysis of the BAL fluid for neutrophil enumeration (Figure 6f). The results show that there were significantly lesser Gr-1/CD11b-positive neutrophils ( $\sim 1.8$-fold) in the BAL of mice infected with AB8879 in comparison with $\mathrm{AB}-19606$. Collectively, these results clearly demonstrate that $\mathrm{AB}-8879$ recruits lesser neutrophils to the lungs early on during infection in comparison with $A B-$ 19606. The observed early reduction in the number of neutrophil/delay in early neutrophil recruitment to the lungs could thus potentially account for the higher bacterial load and subsequent enhanced inflammasome activation exhibited by the clinical isolate AB-8879.

Chemoattractants such as GRO $\alpha$ (KC or CXCL1), MIP-2 (CXCL2), and CXCL5 are important mediators of neutrophil recruitment to the lungs during infection. ${ }^{12,34-37}$ Thus, we next assessed whether the presence of lesser neutrophil numbers in the lungs of mice infected with $\mathrm{AB}-8879$ in comparison with AB-19606 (during early time points of infection) is due to their differential ability to elicit neutrophil chemoattractant production. However, we found that there was no impairment in the production of any of the tested chemokines (GRO $\alpha$, MIP-2, and CXCL5) in the BAL of AB-8879 in comparison with AB-19606 both at 4 and $24 \mathrm{~h}$ after infection (Supplementary Figure S5a-c, respectively). IL-12 is one of the key cytokines reported to be involved in neutrophil accumulation in the lungs during bacterial infections. ${ }^{38}$ Additionally, virulent pathogens such as Francisella tularensis is known to inhibit IL-12 production in the lung to promote infection. ${ }^{39,40}$ Hence, we investigated if AB-8879 differentially regulates IL- 12 production and thereby affect the neutrophil numbers. As shown in Supplementary Figure S5d, both AB-8879 and AB-19606 elicited comparable levels of IL-12p70 in the BAL at both 4 and $24 \mathrm{~h}$ after infection. Collectively, these data suggest that the defect in the neutrophil recruitment/numbers observed during infection with $\mathrm{AB}-8879$ is unlikely due to the defect in the secretion of chemoattractants.

\section{NLRP3 inflammasome machinery contributes to the host defense to clinical isolate, but is dispensable for protection against the type strain of $A$. baumannii}

We next assessed the role of NLRP3 inflammasomes in the host defense to A. baumannii infection. WT or Nlrp3 $3^{-1-}$ mice were intranasally infected with the clinical isolate AB-8879 or with type strain $19606\left(5 \times 10^{7} \mathrm{CFU}\right)$. The lungs were isolated at $24 \mathrm{~h}$ after infection and bacterial load was determined. WT and Nlrp3 $3^{-1-}$ mice infected with AB-19606 strain had comparable bacterial load in the lungs (Figure 7a), possibly due to only minimal inflammasome activation by the type strain (Figure 4a,b). However, Nlrp3 $3^{-1-}$ mice infected with AB8879 had significantly higher bacterial burden in the lungs at $24 \mathrm{~h}$ after infection as compared with the AB-8879-infected WT mice (2.2-fold difference, $P=0.0001$ ) (Figure 7a). This was also associated with a similar increase in bacterial load in the spleens of Nlrp3 ${ }^{-1-}$ mice infected with AB-8879 (1.5-fold difference, $P=0.002$ ) (Figure $7 \mathbf{b}$ ), indicating that NLRP3 may contribute to limit the extrapulmonary dissemination of AB-8879. Therefore, these results clearly demonstrate that whereas NLRP3 pathway is not required for the host defense to 

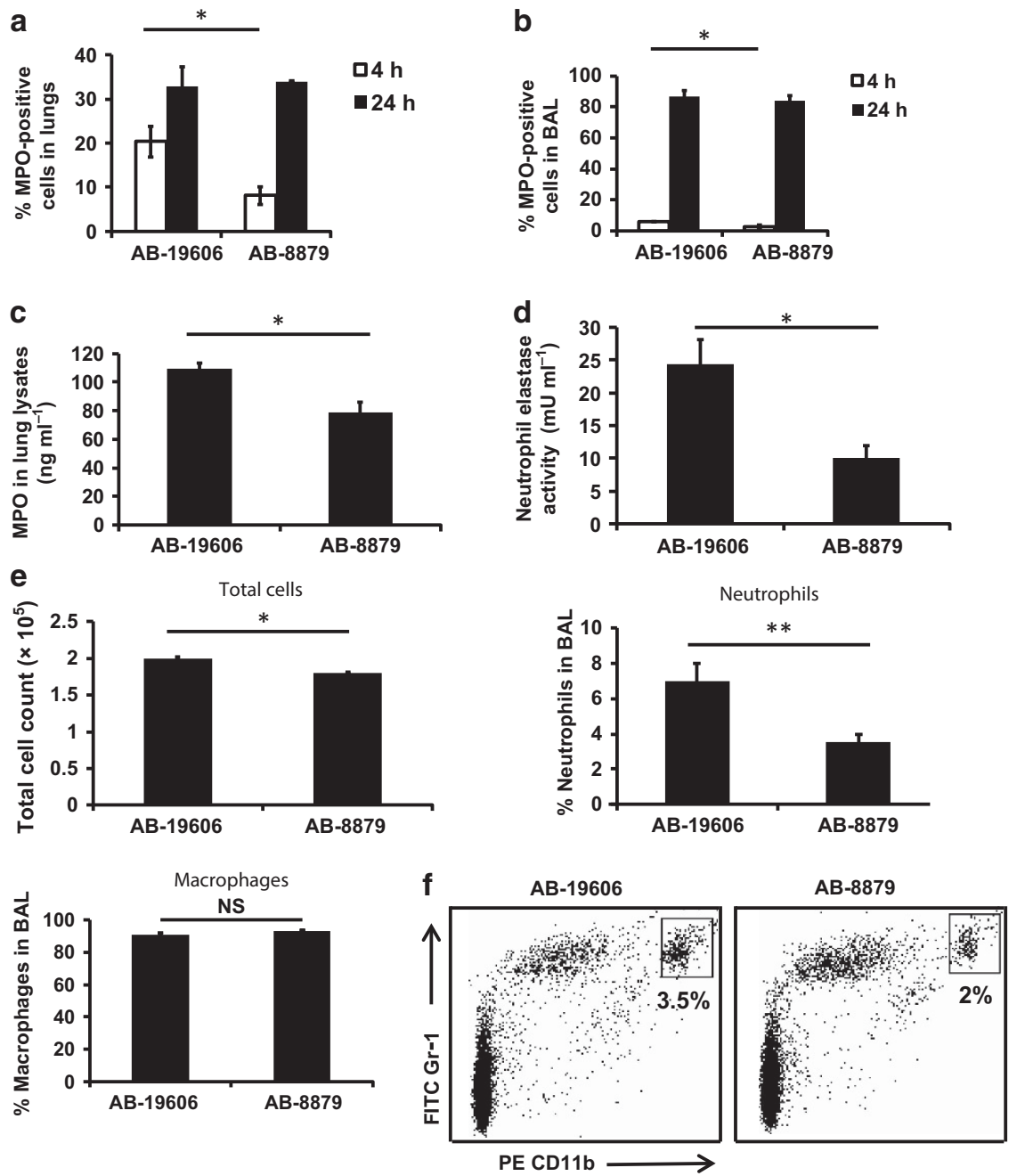

Figure 6 A. baumanniiclinical isolate exhibits lesser early neutrophil influx in the lungs as compared with the type strain. Wild-type C57BL/6 mice were infected with $5 \times 10^{7}$ colony-forming unit (CFU) of $A$. baumannii 19606 (AB-19606) or 8879 (AB-8879) $(n=3-5$ mice per group). At 4 and $24 \mathrm{~h}$ after infection, mice were euthanized. (a) Lungs or (b) bronchoalveolar lavage (BAL) were processed for immunohistochemistry/immunocytochemistry (IHC/ ICC) staining for myeloperoxidase (MPO) as described in the Methods section. (c) MPO levels were determined in the lung lysates obtained at $4 \mathrm{~h}$ after infection by enzyme-linked immunosorbent assay (ELISA). (d) Neutrophil elastase activity was assessed in BAL obtained $4 \mathrm{~h}$ after infection. (e) Cells from BAL obtained at $4 \mathrm{~h}$ after infection were stained with Giemsa and enumerated for total number of cells, neutrophils, or macrophages as described in the Methods section. (f) BAL cells from infected mice at $4 \mathrm{~h}$ after infection were stained with phycoerythrin (PE) CD11b or fluorescein isothiocyanate (FITC) Gr-1 antibodies as described under the Methods section. FACS analysis was performed on Fortessa FACS analyzer. Data are representative of three independent experiments. ${ }^{\star} P \leq 0.05 ;{ }^{* \star} P \leq 0.01$. Values shown represent mean \pm s.d. of results of three independent experiments. NS, not significant.

AB-19606 infection, NLRP3 inflammasome pathway has an important role in the pulmonary host defense to AB-8879 infection.

\section{NLRP3 inflammasome is needed for neutrophil recruitment to the lungs at late stages of infection with $A$. baumannii clinical isolate}

IL-1 $\beta$ is a potent chemoattractant for neutrophils. ${ }^{41} \mathrm{We}$ hypothesized that higher bacterial burden in AB-8879-infected Nlrp3 $3^{-1-}$ mice (Figure 7) might be due to reduced IL-1 $\beta$ production (Figure 4d) and hence reduced neutrophil influx in the lungs of these mice. Hence, we ascertained neutrophil numbers in the lungs/BAL from WT or Nlrp3 ${ }^{-1-}$ mice at $4 \mathrm{~h}$ (early time point) and $24 \mathrm{~h}$ (late time point) after infection. No difference in neutrophils was observed between WT and
$\mathrm{Nlrp}^{-/-}$mice at $4 \mathrm{~h}$ after infection (Figure 8a,b), which was in accordance with the production of only very low levels of detectable IL- $1 \beta$ at this time point. However, by $24 \mathrm{~h}$ after infection, AB-8879 induced IL- $1 \beta$ production in WT mice but not in Nlrp3 $3^{-1-}$ mice (Figure 4d). At this time point, 1.8-fold lesser neutrophils were present in the lungs $(P=0.01)$ and 1.3 -fold lesser neutrophils were present in the BAL $(P=0.007)$ of Nlrp3 $3^{-1-}$ mice as compared with WT mice (Figure 8a,b). Thus, increased susceptibility of Nlrp3 $3^{-1-}$ mice to AB-8879 infection correlates with the presence of lesser neutrophil numbers in the lungs at $24 \mathrm{~h}$ after infection. These results suggest that NLRP3 contributes to the neutrophil recruitment to the lungs during the late stages of infection $(\geq 24 \mathrm{~h}$ after infection) leading to the control of A. baumannii clinical isolate. 


\section{Enhanced NLRP3-dependent production of IL-1//LL-18 in vivo is manifested by $A$. baumannii from different clonal groups}

Having conclusively established that the clinical isolate AB8879 (belonging to ICII) activates the NLRP3 inflammasome in vivo, we next wanted to assess whether other virulent clinical isolates belonging to A. baumannii ICI or ICII are also capable of doing so. For investigating whether the enhanced
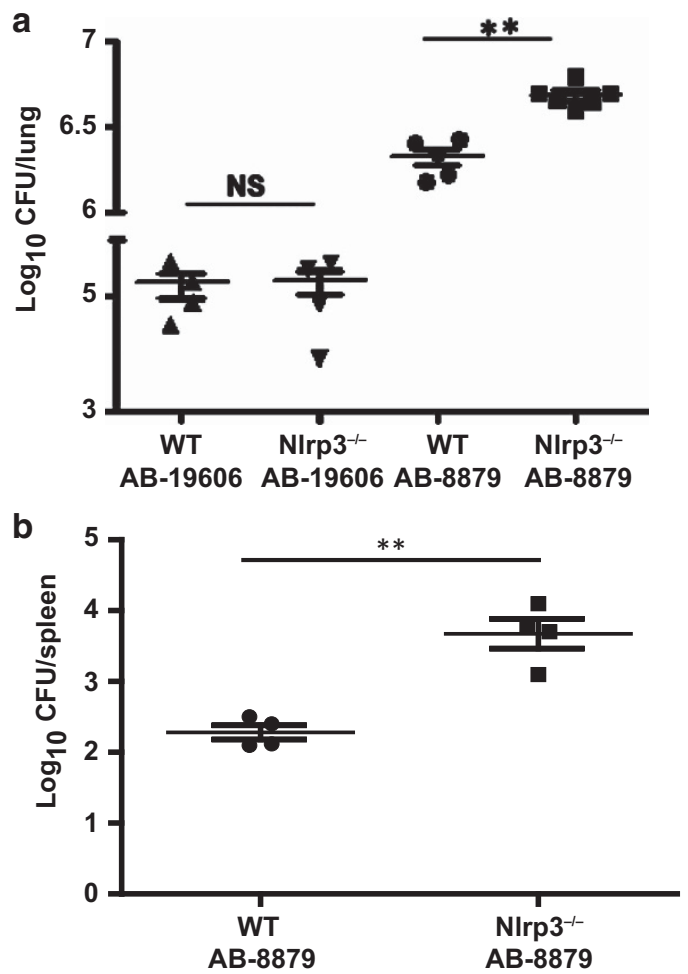

Figure 7 NLRP3 inflammasome machinery contributes to the host defense to clinical isolate, but is dispensable for protection against the type strain of $A$. baumannii. Wild-type (WT) or Nlrp3 ${ }^{-1-}$ mice were infected with $5 \times 10^{7}$ colony-forming unit (CFU) of $A$. baumannii 19606 (AB-19606) or 8879 (AB-8879) ( $n=4-6$ mice per group). At $24 \mathrm{~h}$ after infection, the bacterial load was enumerated in (a) lungs or (b) spleen. ${ }^{*} P \leq 0.05$; ${ }^{* *} P \leq 0.01$. Data are represented as mean \pm s.d. Data are representative of three independent experiments with similar results. NS, not significant. inflammasome activation by AB-8879 in vivo is a conserved phenomenon, we used another XDR-clinical isolate, A. baumannii 40 (AB-40), belonging to IC clonal group $\mathrm{I}^{33}$ as a representative candidate. We found that the $\mathrm{AB}-40$ was also capable of inducing enhanced IL- $1 \beta$ and IL- 18 release in BAL of mice, the levels of which were comparable to those produced by the AB-8879 (Figure 9a,b). Additionally, the production of IL- $1 \beta$ and IL-18 was abrogated in the lungs of AB-40-infected Nlrp3 $3^{-1-}$ mice. This result suggests that enhanced inflammasome activation in vivo may be a common feature of virulent clinical isolates of $A$. baumannii belonging to both ICI and II.

\section{DISCUSSION}

Although the epidemiology and antibiotic resistance traits of A. baumannii clonal groups are well studied, the pathogenesis mechanisms and what determines the clinical success of ICI and II clones are not studied yet in detail. Our study is the first report to demonstrate that clinically relevant $A$. baumannii impairs pulmonary neutrophil recruitment, which may potentially contribute to their clinical success such as higher pulmonary bacterial load and persistence. Our study has further demonstrated that clinical isolates of $A$. baumannii differentially activates the host innate immune NLRP3 inflammasome pathway in comparison with type strain, which could potentially account for the uncontrolled inflammation and lung injury seen in A. baumannii patients with compromised immune system. Our study thus revealed a previously unappreciated role for NLRP3 inflammasome pathway in the host pulmonary defense to A. baumannii clinical isolate.

Early neutrophil recruitment to the lungs was previously shown to be critical for A. baumannii clearance and depletion of neutrophils was associated with higher bacterial load, extrapulmonary dissemination and severe disease in the infected mice. ${ }^{11,14,15}$ Our study demonstrates a novel finding that the clinical isolate AB-8879 belonging to ICII evades this critical step controlling A. baumannii pneumonia by reducing the early neutrophil recruitment to the lungs in comparison with the type strain. This differential interaction with the neutrophils could potentially account for the higher lung bacterial load,
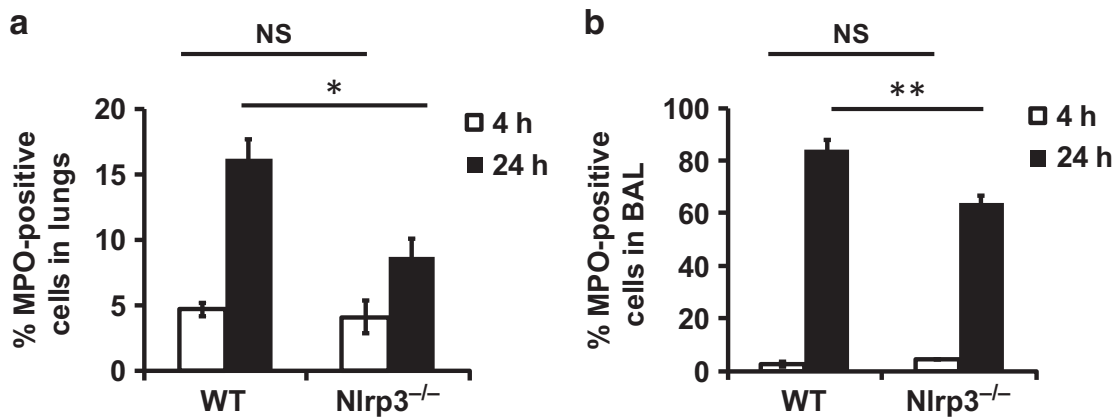

Figure 8 NLRP3 inflammasome promotes neutrophil recruitment to lungs at late stages of infection during infection with $A$. baumannii clinical isolate. Wild-type (WT) or Nlrp3 ${ }^{-1-}$ mice were infected with $5 \times 10^{7}$ colony-forming unit (CFU) of $A$. baumannii-8879 (AB-8879). At 4 or $24 \mathrm{~h}$ after infection, (a) lungs and (b) bronchoalveolar lavage (BAL) were processed for immunohistochemistry/immunocytochemistry (IHC/ICC) staining for myeloperoxidase (MPO). ${ }^{*} P \leq 0.05 ;{ }^{* \star} P \leq 0.01$. Values shown represent mean \pm s.d. and are representative of two independent experiments. 

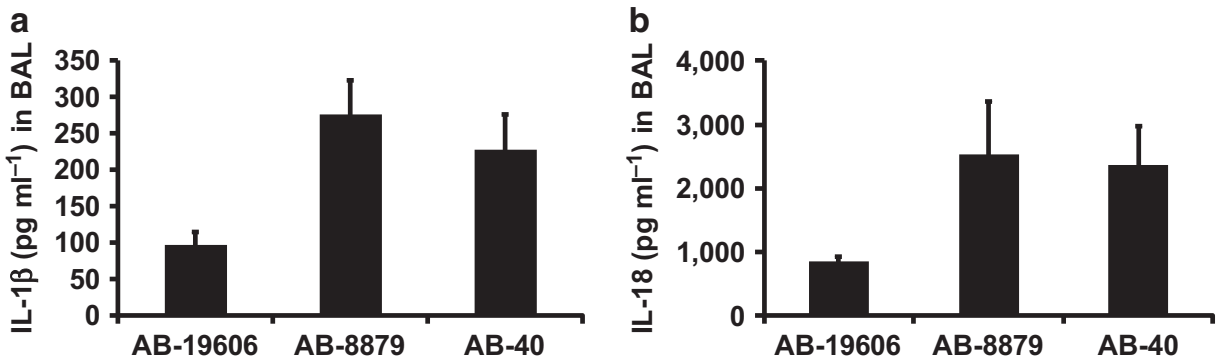

Figure 9 A. baumannii clinical isolate 40 (AB-40) belonging to international clone I (ICI) activates the inflammasome pathway to the same levels as AB-8879 in vivo. Wild-type (WT) mice were infected with $5 \times 10^{7}$ colony-forming unit (CFU) of $A$. baumannii-19606 (AB-19606), 8879 (AB-8879) or 40 (AB-40) isolates ( $n=5$ mice per group). At $24 \mathrm{~h}$ after infection, mice were euthanized and bronchoalveolar lavage (BAL) was obtained and the levels of cytokines IL-1 $\beta$ (a) and IL-18 (b) were analyzed in BAL by luminex assay. Values shown represent mean $\pm \mathrm{s}$.d. and are representative of two independent experiments with similar results.

persistence and extrapulmonary dissemination displayed by the clinical isolate belonging to ICII. Such delayed/reduced neutrophil recruitment to the lungs and enhanced replication of bacteria has been previously reported for virulent bacterial pathogens such as Yersinia pestis. ${ }^{42}$ Yet another study has shown that $\mathrm{A} / \mathrm{J}$ mice were more susceptible to A. baumannii pulmonary infection compared with $\mathrm{B} 6$ mice because $\mathrm{A} / \mathrm{J}$ mice exhibited delayed early recruitment of neutrophils to the lungs that led to higher bacterial burden even at $72 \mathrm{~h}$ after infection. ${ }^{13}$ Thus, apart from further highlighting the importance of early lung neutrophil recruitment in the control of A. baumannii pneumonia, our study also suggests that impairment of early neutrophil recruitment might be a virulence-related trait of A. baumannii clinical isolates. Furthermore, we also show that at least one of the consequences of this delayed neutrophil recruitment and higher bacterial load could be the differential ability of the clinical isolate to activate NLRP3-dependent IL-1 $\beta /$ IL-18 production. Indeed, a previous report showed that higher BAL IL- $1 \beta$ correlated with higher bacterial burden in the lung alveoli of $A$. baumannii pneumonia patients. ${ }^{21}$ The authors further went on to conclude that higher BAL IL-1 $\beta$ levels could be a marker of ongoing inflammation because of persistent bacterial infection despite antibiotic therapy. Thus, our study could also mechanistically explain the correlation of higher IL- $1 \beta$ levels with bacterial persistence and inflammation in patients having virulent clinically relevant $A$. baumannii infections. We therefore propose that inflammasome activation might be a second-line of host defense to clinically relevant virulent A. baumannii that evades the initial neutrophilmediated defense mechanisms, although further studies are warranted to confirm this. On the other hand, it is also possible that enhanced bacterial burden alone cannot simply explain for the heightened inflammasome activation exhibited by AB8879 , but rather the virulence attributes (such as bacterialderived secreted virulence factors, toxins, etc.) or antibiotic resistance pattern of the clinical isolates could also have a role in the enhanced activation of inflammasome pathway. To shed some light into this matter, we performed a comparative genomic analysis of AB-19606 with AB-8879 (Table 1 shows the general features of each of the strains). We found that one of the major differences between the strains is the presence of multiple antibiotic resistance genes in AB-8879 in comparison with AB-19606 (17 genes and 7 genes in AB-8879 and AB-19606, respectively). We further focused on putative genomic islands (Supplementary Figure S6a,b) and virulence genes (Table 2 and Supplementary Figure S6c). Our analysis found that several virulence genes belonging to gene categories such as capsule, and heme utilization were unique to AB-8879 (Table 2). Supplementary Figure S6c summarizes the number of virulence genes found only in AB-19606 or AB-8879 and also the number of genes that are common to both strains. The functional significance of these unique genes in $\mathrm{AB}-8879$ and whether or how they contribute to the differential activation of inflammasome pathway is a topic of future research. In this context, it is also worth noting that although clinical isolate elicited enhanced activation of inflammasome pathway in comparison to type strain in vivo, both clinical and type strains activated inflammasome pathway to comparable levels in BMDMs ex vivo. This result suggests that the differential interaction of the clinical isolate with the immune system may have a major role in the enhanced inflammasome activation elicited by the clinical isolate in vivo. There is also a possibility that the clinical isolate secretes/ produces virulence factors in vivo, but not in vitro, which subsequently activates the inflammasome pathway differentially.

The recruitment of neutrophils to the lung during infection is a multistep process coordinated by the production of chemoattractants, and expression of adhesion molecules by endothelial cells near the site of inflammation/infection. ${ }^{43}$ As we found that there was no significant difference in the BAL levels of chemoattractants such as GRO $\alpha$, MIP-2, CXCL5, and IL-12p70 among AB-8879 and AB-19606, we reason that AB-8879 impairs neutrophil recruitment targeting one or more steps other than the inhibition of chemoattractant production.

A. baumannii infection induces lung inflammation and cell death, and this was proposed to account for the lung injury observed in experimental murine models and patients with A. baumannii pneumonia. ${ }^{44,45}$ Higher levels of LDH observed in the lungs of AB-8879-infected mice in our study may indicate 
Table 1 Summary of general features in AB-8879 and AB-19606 genome

\begin{tabular}{|c|c|c|}
\hline & AB-8879 & AB-19606 \\
\hline Genome size (bp) & $3,848,467^{\mathrm{a}}$ & $3,953,621$ \\
\hline GC content (\%) & $39^{\mathrm{b}}$ & 39.1 \\
\hline Number of plasmid & NA & 1 \\
\hline Number of antibiotic resistance genes (identified by SRST2) & 17 & 7 \\
\hline Genome annotation method & PROKKA 1.12-beta & Ergatis-based workflow \\
\hline Number of protein coding genes & 3,857 & 3,750 \\
\hline Number of rRNA & 3 & 7 \\
\hline Number of tRNA & 62 & 61 \\
\hline Number of tmRNA & 1 & - \\
\hline
\end{tabular}

Table 2 Unique VF identified in AB-19606 and AB-8879

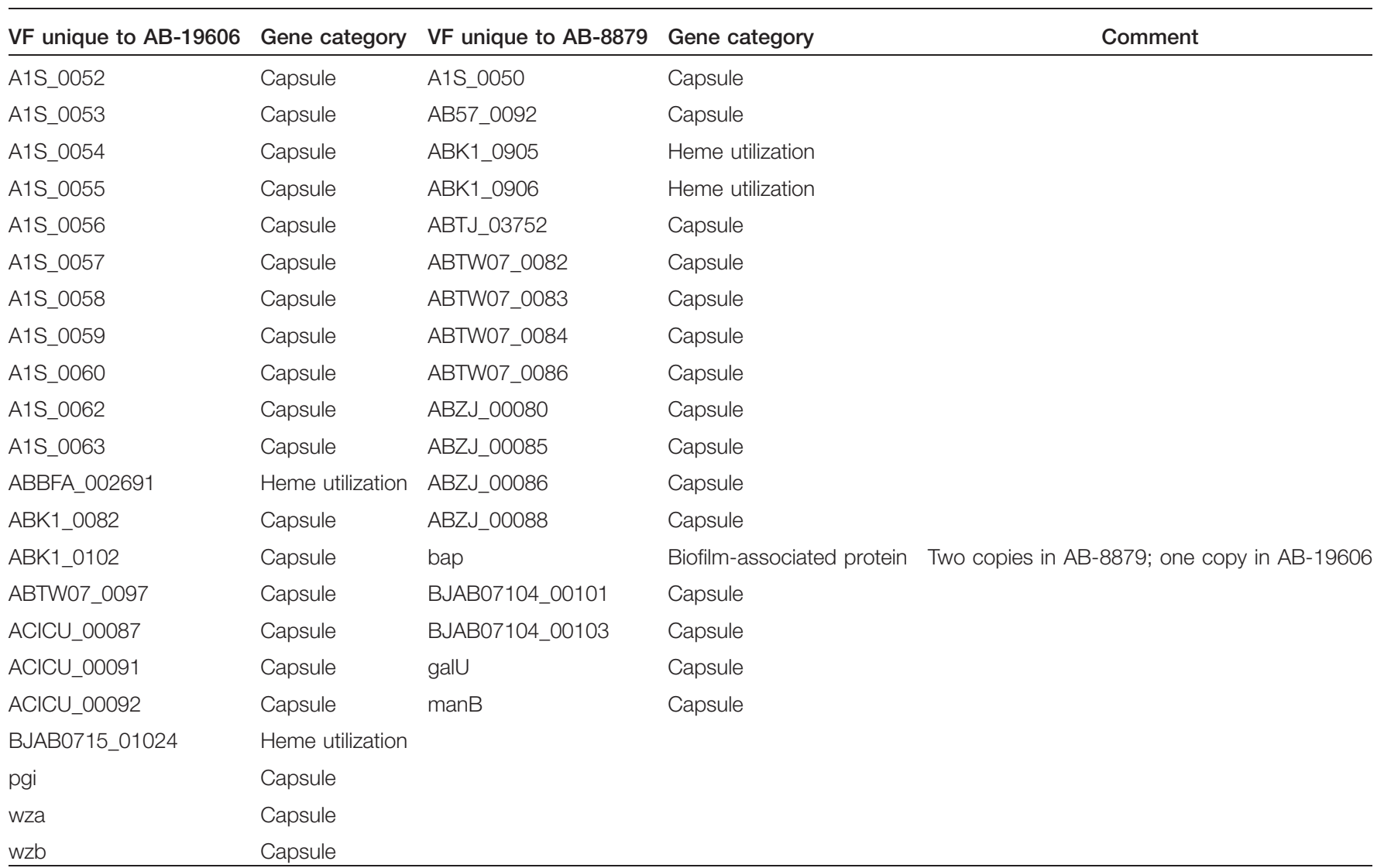

$\mathrm{VF}$, virulence factor.

that the clinical isolate induces more cell death/lung damage in comparison with the type strain. In addition, the cell death induced by $\mathrm{AB}-8879$ was reduced in Nlrp3 ${ }^{-1-}$ mice implying that NLRP3 pathway contributes to the cell death/lung damage during A. baumannii infection. We also found that the IL-1 $\beta /$ IL-18 production during $A$. baumannii infection occurs via cell 
death inducing caspase-1 and caspase 11-dependent pathways. Caspase- 11 is associated with the noncanonical inflammasome pathway that is involved in NLRP3-dependent activation of caspase-1, release of IL- $1 \beta / \mathrm{IL}-1 \alpha$, and inflammatory cell death (pyroptosis) in response to various Gram-negative bacterial pathogens. ${ }^{32,46,47}$ It will be important to tease out the relative contributions of caspase- 1 and caspase-11 in the induction of pyroptosis during clinically relevant $A$. baumannii infections.

Our study revealed that $\mathrm{AB}-8879$ caused enhanced activation of NLRP3 inflammasome pathway in comparison with AB-19606. We also demonstrate that, besides contributing to cell death/lung damage, NLRP3 inflammasome is also required for the pulmonary host defense against the clinical isolate AB-8879, but not AB-19606. Thus, inflammasome pathway may likely have a dual role (either protective or deleterious or both) in A. baumannii infection depending on the immune status of the host and also virulence of the bacteria. In fact, most $A$. baumannii infections occur in patients with underlying disease conditions or compromised immune status, suggesting that variability in the host immune response may contribute to the risk or severity of infection. Assessing the role of NLRP3 inflammasome in neutropenic mouse infection models (that resembles the immunocompromised condition) will be helpful to clarify this point further. Our study showed that another clinical isolate belonging to ICI (AB-40) also activates NLRP3-inflammasome-dependent IL-1 $\beta /$ IL-18 to the same level as AB-8879. Thus, our experiments provide reasonable evidence to suggest that heightened inflammasome activation is a common feature of clinically relevant A. baumannii isolates. Further studies using additional isolates belonging to ICI and II will be needed to determine the wider occurrence of clone/strain/isolate-specific inflammasome response to A. baumannii virulent infections.

Important role of IL-1-associated pathways in pneumonia has been documented for various bacterial infections. ${ }^{48}$ The effects of IL-1 are tightly controlled by two naturally occurring inhibitors, antagonist of IL-1 receptor and IL-1R type II (IL-1RII). Antagonist of IL-1 receptor is an anti-inflammatory protein that counteracts the uncontrolled effects of proinflammatory responses to reduce the destructive immunopathology. Indeed, several studies have shown that polymorphism of antagonist of IL-1 receptor gene is associated with respiratory injury/pneumonia in patients. Some examples includes: reduced levels of IL-1RA have been identified as a marker for pneumonia in the elderly patients; ${ }^{49}$ genetic polymorphism of IL-1RA pathway is associated with pathophysiology of invasive pneumococcal disease in patients; ${ }^{50}$ Variable number tandem repeat polymorphism within the intron 2 of $I L$ - $1 \mathrm{ra}$ gene was associated with respiratory injury in children who had community-acquired pneumonia, ${ }^{51}$ etc. More importantly, a previous study suggested that IL-1RA polymorphism is associated with patients with multidrugresistant- $A$. baumannii-related pneumonia. ${ }^{22}$ Thus, the expression levels/polymorphism of IL-1-associated pathway molecules could be considered as a marker for disease severity in A. baumannii pneumonia patients.
Collectively, our study demonstrates the importance of NLRP3 inflammasome pathway in the immunity and pathogenesis of clinically relevant $A$. baumannii infections. This study also opens the possibility that therapies targeted at modulating inflammasome pathway could be considered as a viable option for either promoting bacterial control or for ameliorating the pathologic consequences associated with virulent clinically relevant $A$. baumannii infections.

\section{METHODS}

Ethics statement. All animal experiments were performed in strict accordance with the prevailing Singapore National Advisory Committee for Laboratory Animal Research (NACLAR) guidelines and approved by Sing-Health Institutional Animal Care and Use Committee, Singapore (Protocol No.: 2013/SHS/863). NACLAR was established to develop national guidelines for the care and use of animals for scientific purposes in Singapore.

Mice. Eight-twelve-week-old female mice were used for all the mice infection experiments. C57BL/6J WT mice were purchased from the Biological Resource Center (Agency for Science, Technology, and Research (A $\left.{ }^{\star} \mathrm{STAR}\right)$, Singapore). Nlrp3 ${ }^{-1-}, 52 \mathrm{Asc}^{-1-}, 53$ Casp-1 ${ }^{-1-}, 54$ Casp-11 $11^{-1-54}$ and Casp- $1 / 11^{-/-55}$ mice were used for the experiments. All mutants were backcrossed to C57BL/6 background for at least 10 generations.

Reagents and cell treatment. Tryptic soy broth (22092), tryptic soy agar (22091) and polymyxin B (P4932) were purchased from SigmaAldrich (St. Louis, MO). LDH Cytotoxicity Kit (88953) was obtained from Pierce (Rockford, IL). LPS (EK-3701) was purchased from Invivogen (San Diego, CA) and was used at $100 \mathrm{ng} \mathrm{ml}^{-1}$ where indicated. ATP (A2383) was purchased from Sigma-Aldrich and was used at $5 \mathrm{~mm}$ where indicated. ELISA Kits for IL-1 $\beta$ (BMS6002TWO), IL-18 (BMS618/3), and tumor necrosis factor- $\alpha$ (BMS607HS) were purchased from e-Bioscience (San Diego, CA). MIP-2 ELISA Kit (SMM200) was purchased from R\&D (Minneapolis, MN). Mouse IL-1 $\alpha$ ELISA Kit (ab113344) was obtained from Abcam (Cambridge, MA). Caspase-1 antibody (AG-20B-0042-C100) was obtained from AdipoGen Life Sciences (San Diego, CA) and was used at 1:1,000 dilution for western blot. Neutrophil Elastase Activity Kit was purchased from Cayman Chemical (Ann Arbor, MI) (600610). MPO ELISA Kit (ab155458) was purchased from Abcam. MPO antibody (Ab-9535) was purchased from Abcam and was used at 1:25 dilution for immunohistochemistry (IHC) and immunocytochemistry (ICC). WrightGiemsa stain (WG128) was purchased from Sigma-Aldrich. Lipofectamine 3000 (L3000015) was obtained from Thermo Fisher Scientific (Waltham, MA) and was used as per the manufacturer's instructions. Luciferase Kit (E1910) was obtained from Promega (Madison, WI). ATP ELISA Kit (ABIN627439) was purchased through antibodies-online.com. All kits were used as per the manufacturer's instructions.

CA-074 Me (BML-P1126-0001) was purchased from Enzo Life Sciences (Farmingdale, NY). APDC (sc-202408A) was purchased from Santa Cruz (Dallas, TX), KCl (P5405) and cytochalasin D (C8273) from Sigma-Aldrich. The reagents were used at following concentrations: CA-074 Me, $25 \mu \mathrm{M}$; APDC, $100 \mu \mathrm{M} ; \mathrm{KCl}, 50 \mathrm{~mm}$; cytochalasin D, $5 \mu \mathrm{M}$. The cells were treated with these reagents for $1 \mathrm{~h}$ before infection and were maintained in these reagents throughout the course of the experiments.

Bacterial strains and growth conditions. $\mathrm{AB}-8879$ and $\mathrm{AB}-40$ isolates were cultured from skin tissue specimen and blood specimen of a burns patient, respectively. ${ }^{26,27,33}$ Both AB-8879 and AB-40 were $\mathrm{XDR}$, and susceptible to only polymyxin B or tigecycline. A. baumannii ATCC 19606, AB-8879, and AB-40 strains were grown overnight in Tryptic soy broth supplemented with $25 \mu \mathrm{g} \mathrm{ml}^{-1}$ streptomycin at $37^{\circ} \mathrm{C}$ with shaking at 220 r.p.m. 
Generation of BMDM. BMDMs from indicated mice were isolated and cultured as described before. ${ }^{17}$ Briefly, bone marrow was collected from the femur and tibia of 6-8-week-old mice. The bone marrow cells were cultured in Dulbecco's modified Eagle's medium (Gibco, Waltham, MA) supplemented with $10 \%$ fetal calf serum (Gibco), penicillin-streptomycin-amphoterecin (Thermo Fisher Scientific) and 30\% L929 cell culture supernatants containing murine macrophage colony-stimulating factor. At day 7 , nonadherent cells were washed off and the adherent macrophages were removed using cell scrapers and used for further experiments.

In vitro A. baumannii infection. BMDMs $\left(0.5 \times 10^{6}\right)$ from indicated mice were seeded in a 24 -well plate. Cells were pretreated with $100 \mathrm{ng} \mathrm{ml}^{-1}$ LPS overnight where mentioned. The cells were washed with phosphate-buffered saline (PBS) before infection. Overnight grown cultures of $A$. baumannii were harvested by centrifugation $(3,000 \mathrm{~g}, 10 \mathrm{~min})$, washed two times with PBS, and finally resuspended in Dulbecco's modified Eagle's medium containing $10 \%$ fetal bovine serum medium. Cells were infected at MOI 100. To synchronize entry, the bacteria were centrifuged at $600 \mathrm{~g}$ for $5 \mathrm{~min}$ and were incubated with cells for $2 \mathrm{~h}$. Subsequently, the cells were washed three times with PBS. Cells were then treated with polymyxin B $\left(50 \mu \mathrm{g} \mathrm{ml}^{-1}\right)$ for $1 \mathrm{~h}$ to kill the extracellular bacteria. Cells were washed three times with PBS and either lysed in PBS containing $0.1 \%$ Triton X-100 and plated on trypticase soy agar (TSA) streptomycin plates to assess cell invasion (by calculating the CFU) or maintained in medium with $5 \mu \mathrm{g} \mathrm{ml}^{-1}$ of polymyxin $\mathrm{B}$ for the rest of the duration of the experiment.

In vivo A. baumannii infection. Mice were infected with $A$. baumannii (AB-8879 or AB-19606) intranasally as described earlier. ${ }^{15}$ Briefly, bacteria were grown overnight in tryptic soy broth supplemented with $25 \mu \mathrm{g} \mathrm{ml}^{-1}$ streptomycin. Next morning the cultures were reinoculated in tryptic soy broth at $1 \%$. After $3 \mathrm{~h}$, the bacteria were washed and processed as described in the previous section. The bacteria were resuspended at indicated CFUs in $40 \mu \mathrm{l}$ PBS. Actual inoculum concentrations were determined by plating serial dilutions on TSA plates supplemented with $25 \mu \mathrm{g} \mathrm{ml}^{-1}$ streptomycin. Mice were anesthetized by administering ketamine/xylazine intraperitoneally and were subsequently infected intranasally with A.baumannii.

In vivo bacterial load enumeration. For enumeration of bacterial CFU in various organs, mice were killed at indicated time points after infections. Various organs were removed aseptically and were homogenized in $0.1 \%$ Triton X-100. Serial dilutions of the lysates were plated on TSA streptomycin plates for CFU enumeration. For enumeration of bacterial CFU in blood, blood was collected from the submandibular vein in EDTA-coated tubes. Serial dilutions of blood were plated on TSA streptomycin plates to enumerate bacteria.

Collection of BAL. For the aspiration of BAL, trachea was exposed through a midline incision. Lungs were lavaged 4-5 times with $1 \mathrm{ml}$ PBS injected through the trachea, as described previously. ${ }^{15}$ BAL fluid was spun at 2,000 r.p.m. for $10 \mathrm{~min}$. The cells were processed for IHC, ICC and Giemsa staining as described below. The supernatant was frozen at $-80^{\circ} \mathrm{C}$ and was analyzed for various cytokines as described below.

Giemsa staining, IHC and ICC. BAL was spun at 2,000 r.p.m. for $10 \mathrm{~min}$. The cell pellet was resuspended in PBS-bovine serum albumin $1 \%$ and was spun onto Superfrost Plus Slides (J1800AMN2; Thermo Fisher Scientific) using Cytospin 3 centrifuge. Slides were prepared for ICC or for Giemsa staining. ICC staining for MPO analysis was carried out in the histopathology laboratory at The Advanced Molecular Pathology Laboratory (AMPL), Institute of Molecular and Cell Biology (IMCB), Singapore.

IHC staining of MPO in lungs was carried out as follows. Lungs were insufflated with $10 \%$ neutral-buffered formalin via trachea and removed en bloc. The lungs were kept in $10 \%$ neutral buffer formalin until further processing for histological studies, which was carried out in the histopathology laboratory at AMPL, IMCB Singapore as described above. Lungs were embedded in paraffin blocks. Serial sections $(4-5 \mu \mathrm{m})$ were cut and processed for MPO staining by IHC. ICC and IHC slides were scanned at $\times 20$ using Leica SCN400 Slide Scanner (Leica Microsystems, Wetzlar, Germany). Images were exported to Slidepath Digital Image Hub (Leica Microsystems) for viewing. Images were analyzed using the Slidepath Tissue IA 2.0 Software (Leica Microsystems). Data were collated using Microsoft Excel. Giemsa-stained slides were examined under light microscope at $\times 100$ for differential cell counts. Hundred cells per slide were counted and the percentage of neutrophils and macrophages was determined.

Cytokine analysis. For the in vitro experiments, cell culture supernatants were collected at different time points after infection. The cytokines were analyzed using ELISA Kits according to manufacturer's instructions. For the analysis of cytokines produced during in vivo infection, BAL was collected from mice at various time points after infection, as already described. The lavage was spun at 2,000 r.p.m. for $10 \mathrm{~min}$. The supernatant was concentrated using Vivaspin $500 \mathrm{col}-$ umns ( $5 \mathrm{kDa}$ cutoff, VS0012; Sartorius, Gottingen, Germany). The supernatant was analyzed for various cytokines using ProcartaPlex Multiplex Immunoassay from eBioscience by the Immunomonitoring Platform, SIgN, A*STAR, Singapore.

For the analysis of MPO by ELISA, lungs from mice were obtained at indicated time points and homogenized in $0.1 \%$ Triton X-100. The lysates were then spun at 2,000 r.p.m. for $10 \mathrm{~min}$. The supernatants were frozen at $-80^{\circ} \mathrm{C}$ and were later analyzed for MPO by ELISA as per the manufacturer's instructions.

RNA isolation and quantitative reverse transcription-PCR. At designated time points, BMDMs were lysed in RLT buffer and RNA was isolated using RNeasy Kit (74106; Qiagen). cDNA synthesis was carried out by iScript cDNA Synthesis Kit (170-8891; Bio-Rad, Hercules, CA) as per the manufacturer's instructions. Primer sequences for various genes amplified were as described in Bouwman et al. ${ }^{56}$ Reactions were performed in Roche Light Cycler 480 Real-time PCR system under the following conditions: $94^{\circ} \mathrm{C} / 2$ min for 1 cycle; $94{ }^{\circ} \mathrm{C} / 30 \mathrm{~s}, 50{ }^{\circ} \mathrm{C} / 30 \mathrm{~s}, 72{ }^{\circ} \mathrm{C} / 60 \mathrm{~s}$ for 40 cycles; $72{ }^{\circ} \mathrm{C} / 7 \mathrm{~min}$ for 1 cycle. Results were analyzed using delta-delta - Ct method.

Western blot. BMDMs $\left(2 \times 10^{6}\right)$ were seeded in a 24 -well plate and infected with A.baumannii as described above. At $24 \mathrm{~h}$ after infection, cell culture supernatants were collected and concentrated using Vivaspin 500 Columns (cutoff $5 \mathrm{kDa}$, VS0012; Sartorius). The concentrated supernatants were separated by $12 \%$ denaturing sodium dodecyl sulfate-polyacrylamide gel electrophoresis. Western blotting was performed on the separated proteins transferred to PVDF membrane using standard procedures. Blots were probed for caspase-1 p20. The signal was developed using an ECL Western Kit (GE Healthcare, Chicago, IL).

FACS staining. Mice were infected with AB-8879 or AB-19606. At $4 \mathrm{~h}$ after infection, mice were killed and BAL was collected on ice. BAL was spun at 1,500 r.p.m. for $10 \mathrm{~min}$ at $4{ }^{\circ} \mathrm{C}$. The cell pellet was resuspended in PBS- bovine serum albumin $1 \%$ and stained with the following antibodies: PE CD11b (130-098-087; Miltenyi Biotec, Bergisch Gladbach, Germany) and FITC Ly6G (130-102-934; Miltenyi Biotec) as per the manufacturer's instructions. The cells were subsequently fixed in $4 \%$ paraformaldehyde. The samples were run on Fortessa FACS analyzer (BD Biosciences, NJ) and the analysis was carried out with the FlowJo Software (FlOWJO, LLC, Ashland, OR).

Genomic analysis. Genome sequence of AB-19606 was obtained from GenBank (accession number JMRY00000000.1). Genome sequence of $\mathrm{AB}-8879$ is available in our local database. Genome annotation was performed using PROKKA on the assembled contigs of AB-8879. The annotated genomes of AB-8879 and AB-19606 in GenBank format were used in IslandViewer3 to predict genomic islands using 
A. baumannii multidrug-resistant-ZJ06 complete genome as reference. SRST2 is used to identify the antibiotic resistance genes found in AB-8879 and AB-19606 using ARGannot resistance gene database included in the program. SRST2 was used to identify virulence genes in A. baumannii AB-8879 and AB-19606. To generate the virulence genes database for SRST2, DNA sequences of full data set was downloaded from the virulence factor database. Then, the virulence genes by A. baumannii were extracted and clustered at $90 \%$ nucleotide identity. SRST2 uses sequencing reads for genes typing. Paired-end reads from Illumina sequencing for AB-8879 is available in our database. Single-ended 100 bp reads from Illumina HiSeq 2000 for AB-19606 was extracted using SRA Toolkit under the accession number SRR2180354 from NCBI.

Statistical analysis. $P$ values were determined by unpaired two-tailed Student's $t$-test. Analysis of variance test was used in multiple group comparisons. $P$ values $<0.05$ were considered statistically significant.

SUPPLEMENTARY MATERIAL is linked to the online version of the paper at http://www.nature.com/mi

\section{ACKNOWLEDGMENTS}

We thank the team of Mutant Mouse Collection Core Service (Singapore Immunology Network, SlgN) for assistance in breeding and husbandry of some mutant mice and Immunomonitoring Platform (SlgN) for luminex analysis. We also thank Dr Ong Twee Hee (National University of Singapore, Singapore) for helping with the genome analysis of the strains. This work was supported by Duke-NUS Signature Research Program funded by Agency for Science Technology and Research (A*STAR) \& Ministry of Health, Singapore (to B.S.); National Medical Research Council Centre Grant (NMRC/CG/016/2013) (to A.L.K.), and Singapore Immunology Network (SlgN) core funds (to A.M.).

\section{AUTHOR CONTRIBUTIONS}

Conceived and designed the experiments: N.D. and B.S. Performed the experiments: N.D., S.D.K., H.J.K., V.B., C.Y.T., P.K. and L.T.P. Analyzed the data: N.D., T.T.T., A.L.K., A.M. and B.S. Contributed reagents/materials: A.M. and A.L.K. Wrote the manuscript: N.D. and B.S. Critically revised the manuscript: A.M. and H.J.K.

\section{DISCLOSURE}

The authors declared no conflict of interest.

c) 2018 Society for Mucosal Immunology

\section{REFERENCES}

1. Antunes, L.C., Visca, P. \& Towner, K.J. Acinetobacter baumannii: evolution of a global pathogen. Pathog. Dis. 71, 292-301 (2014).

2. Falagas, M.E., Karveli, E.A., Kelesidis, I. \& Kelesidis, T. Communityacquired Acinetobacter infections. Eur. J. Clin. Microbiol. Infect. Dis. 26, 857-868 (2007).

3. Fournier, P.E. \& Richet, H. The epidemiology and control of Acinetobacter baumannii in health care facilities. Clin. Infect. Dis. 42, 692-699 (2006).

4. Gaynes, R. \& Edwards, J.R. National Nosocomial Infections Surveillance S. Overview of nosocomial infections caused by Gram-negative bacilli. Clin. Infect. Dis. 41, 848-854 (2005).

5. McConnell, M.J., Actis, L. \& Pachon, J. Acinetobacter baumannii: human infections, factors contributing to pathogenesis and animal models. FEMS Microbiol. Rev. 37, 130-155 (2013).

6. Gordon, N.C. \& Wareham, D.W. Multidrug-resistant Acinetobacter baumannii: mechanisms of virulence and resistance. Int. J. Antimicrob. Agents 35, 219-226 (2010).

7. Cerqueira, G.M. \& Peleg, A.Y. Insights into Acinetobacter baumannii pathogenicity. IUBMB Life 63, 1055-1060 (2011).

8. Pogue, J.M., Mann, T., Barber, K.E. \& Kaye, K.S. Carbapenem-resistant Acinetobacter baumannii: epidemiology, surveillance and management. Expert Rev. Anti-infect. Ther/ 11, 383-393 (2013).
9. Teo, J.Q., Cai, Y., Lim, T.P., Tan, T.T. \& Kwa, A.L. Carbapenem resistance in Gram-negative bacteria: the not-so-little problem in the little red dot. Microorganisms 4, pii: E13 (2016).

10. Bergogne-Berezin, E. \& Towner, K.J. Acinetobacter spp. as nosocomial pathogens: microbiological, clinical, and epidemiological features. Clin. Microbiol. Rev. 9, 148-165 (1996).

11. Breslow, J.M. et al. Innate immune responses to systemic Acinetobacter baumannii infection in mice: neutrophils, but not interleukin-17, mediate host resistance. Infect. Immun. 79, 3317-3327 (2011).

12. Knapp, S. et al. Differential roles of CD14 and toll-like receptors 4 and 2 in murine Acinetobacter pneumonia. Am. J. Respir. Crit. Care Med. 173, 122-129 (2006).

13. Qiu, H., KuoLee, R., Harris, G. \& Chen, W. High susceptibility to respiratory Acinetobacter baumannii infection in $\mathrm{A} / \mathrm{J}$ mice is associated with a delay in early pulmonary recruitment of neutrophils. Microbes Infect./Instit. Pasteur 11, 946-955 (2009).

14. Qui, H., Kuolee, R., Harris, G. \& Chen, W. Role of NADPH phagocyte oxidase in host defense against acute respiratory Acinetobacter baumannii infection in mice. Infect. Immun. 77, 1015-1021 (2009).

15. van Faassen, H., KuoLee, R., Harris, G., Zhao, X., Conlan, J.W. \& Chen, W. Neutrophils play an important role in host resistance to respiratory infection with Acinetobacter baumannii in mice. Infect. Immun. 75, 5597-5608 (2007).

16. Kim, C.H. et al. Tollike receptor 2 promotes bacterial clearance during the initial stage of pulmonary infection with Acinetobacter baumannii. Mol. Med. Rep. 9, 1410-1414 (2014).

17. Bist, P., Dikshit, N., Koh, T.H., Mortellaro, A., Tan, T.T. \& Sukumaran, B. The Nod1, Nod2, and Rip2 axis contributes to host immune defense against intracellular Acinetobacter baumannii infection. Infect. Immun. 82, 1112-1122 (2014).

18. Noto, M.J., Boyd, K.L., Burns, W.J., Varga, M.G., Peek, R.M. Jr. \& Skaar, E.P. Toll-like receptor 9 contributes to defense against Acinetobacter baumannii infection. Infect. Immun. 83, 4134-4141 (2015).

19. de Breij, A. et al. Do biofilm formation and interactions with human cells explain the clinical success of Acinetobacter baumannii?. PLOS ONE 5, e10732 (2010).

20. de Breij, A., Eveillard, M., Dijkshoorn, L., van den Broek, P.J., Nibbering, P.H. \& Joly-Guillou, M.L. Differences in Acinetobacter baumannii strains and host innate immune response determine morbidity and mortality in experimental pneumonia. PLoS ONE 7, e30673 (2012).

21. Wu, C.L. et al. Bronchoalveolar interleukin-1 beta: a marker of bacterial burden in mechanically ventilated patients with community-acquired pneumonia. Crit. Care Med. 31, 812-817 (2003).

22. Hsu, M.J., Lu, Y.C., Hsu, Y.C., Liu, W.S. \& Wu, W.T. Interleukin-1 receptor antagonist gene polymorphism in patients with multidrug-resistant Acinetobacter baumannii-associated pneumonia. Ann. Thorac. Med. 7, 74-77 (2012)

23. Broz, P. \& Dixit, V.M. Inflammasomes: mechanism of assembly, regulation and signalling. Nat. Rev. Immunol. 16, 407-420 (2016).

24. Chaput, C., Sander, L.E., Suttorp, N. \& Opitz, B. NOD-like receptors in lung diseases. Front. Immunol. 4, 393 (2013).

25. Witzenrath, M. et al. The NLRP3 inflammasome is differentially activated by pneumolysin variants and contributes to host defense in pneumococcal pneumonia. J. Immunol. 187, 434-440 (2011).

26. Lim, T.P. et al. In vitro activity of various combinations of antimicrobials against carbapenem-resistant Acinetobacter species in Singapore. J. Antibiot. (Tokyo) 62, 675-679 (2009).

27. Lim, T.P. et al. In vitro activity of polymyxin B, rifampicin, tigecycline alone and in combination against carbapenem-resistant Acinetobacter baumannii in Singapore. PLOS ONE 6, e18485 (2011).

28. Latz, E., Xiao, T.S. \& Stutz, A. Activation and regulation of the inflammasomes. Nat. Rev. Immunol. 13, 397-411 (2013).

29. Mortensen, B.L. \& Skaar, E.P. Host-microbe interactions that shape the pathogenesis of Acinetobacter baumannii infection. Cell. Microbiol. 14, 1336-1344 (2012).

30. Vigano, E. \& Mortellaro, A. Caspase-11: the driving factor for noncanonical inflammasomes. Eur. J. Immunol. 43, 2240-2245 (2013).

31. Rayamajhi, M., Zhang, Y. \& Miao, E.A. Detection of pyroptosis by measuring released lactate dehydrogenase activity. Methods Mol. Biol. 1040, 85-90 (2013). 
32. Kayagaki, N. et al. Noncanonical inflammasome activation by intracellular LPS independent of TLR4. Science 341, 1246-1249 (2013).

33. Lim, T.P. et al. Multiple genetic mutations associated with polymyxin resistance in Acinetobacter baumannii. Antimicrob. Agents Chemother. 59, 7899-7902 (2015).

34. Bozic, C.R. et al. Expression and biologic characterization of the murine chemokine KC. J. Immunol. 154, 6048-6057 (1995).

35. Driscoll, K.E. et al. Cloning, expression, and functional characterization of rat MIP-2: a neutrophil chemoattractant and epithelial cell mitogen. J. Leukoc. Biol. 58, 359-364 (1995).

36. Erridge, C., Moncayo-Nieto, O.L., Morgan, R., Young, M. \& Poxton, I.R. Acinetobacter baumannii lipopolysaccharides are potent stimulators of human monocyte activation via Toll-like receptor 4 signalling. J. Med. Microbiol. 56 (Pt 2), 165-171 (2007).

37. Mei, J. et al. CXCL5 regulates chemokine scavenging and pulmonary host defense to bacterial infection. Immunity 33, 106-117 (2010).

38. Sun, K., Salmon, S.L., Lotz, S.A. \& Metzger, D.W. Interleukin-12 promotes gamma interferon-dependent neutrophil recruitment in the lung and improves protection against respiratory Streptococcus pneumoniae infection. Infect. Immun. 75, 1196-1202 (2007).

39. Bauler, T.J., Chase, J.C. \& Bosio, C.M. IFN-beta mediates suppression of IL-12p40 in human dendritic cells following infection with virulent Francisella tularensis. J. Immunol. 187, 1845-1855 (2011).

40. Ireland, R., Wang, R., Alinger, J.B., Small, P. \& Bosio, C.M. Francisella tularensis SchuS4 and SchuS4 lipids inhibit IL-12p40 in primary human dendritic cells by inhibition of IRF1 and IRF8. J. Immunol. 191, 1276-1286 (2013).

41. Oliveira, S.H., Canetti, C., Ribeiro, R.A. \& Cunha, F.Q. Neutrophil migration induced by IL-1beta depends upon LTB4 released by macrophages and upon TNF-alpha and IL-1beta released by mast cells. Inflammation $\mathbf{3 1}$, 36-46 (2008).

42. Vagima, Y. et al. Circumventing Y. pestis virulence by early recruitment of neutrophils to the lungs during pneumonic plague. PLOS Pathog. 11, e1004893 (2015).

43. Williams, M.R., Azcutia, V., Newton, G., Alcaide, P. \& Luscinskas, F.W. Emerging mechanisms of neutrophil recruitment across endothelium. Trends Immunol. 32, 461-469 (2011).
44. Russo, T.A. et al. Rat pneumonia and soft-tissue infection models for the study of Acinetobacter baumannii biology. Infect. Immun. 76, 3577-3586 (2008).

45. Smani, Y., Docobo-Perez, F., McConnell, M.J. \& Pachon, J. Acinetobacter baumannii-induced lung cell death: role of inflammation, oxidative stress and cytosolic calcium. Microb. Pathog. 50, 224-232 (2011).

46. Broz, P. et al. Caspase-11 increases susceptibility to Salmonella infection in the absence of caspase-1. Nature 490, 288-291 (2012).

47. Rathinam, V.A. et al. TRIF licenses caspase-11-dependent NLRP3 inflammasome activation by Gram-negative bacteria. Cell 150, 606-619 (2012).

48. Sahoo, M., Ceballos-Olvera, I., del Barrio, L. \& Re, F. Role of the inflammasome, IL-1beta, and IL-18 in bacterial infections. Scientific World J. 11, 2037-2050 (2011).

49. Zhao, L., Wang, L., Zhang, X., Sun, L., Sun, D. \& Sun, T. Reduced levels of interleukin1 receptor antagonist act as a marker for pneumonia in the elderly. Mol. Med. Rep. 10, 959-964 (2014).

50. Carrol, E.D. et al. The IL1RN promoter rs4251961 correlates with IL-1 receptor antagonist concentrations in human infection and is differentially regulated by GATA-1. J. Immunol. 186, 2329-2335 (2011).

51. Patwari, P.P. et al. Interleukin-1 receptor antagonist intron 2 variable number of tandem repeats polymorphism and respiratory failure in children with community-acquired pneumonia. Pediatr. Crit. Care Med. 9, 553-559 (2008).

52. Martinon, F., Petrilli, V., Mayor, A., Tardivel, A. \& Tschopp, J. Goutassociated uric acid crystals activate the NALP3 inflammasome. Nature 440, 237-241 (2006).

53. Mariathasan, S. et al. Differential activation of the inflammasome by caspase-1 adaptors ASC and Ipaf. Nature 430, 213-218 (2004).

54. Kayagaki, N. et al. Non-canonical inflammasome activation targets caspase-11. Nature 479, 117-121 (2011).

55. Kuida, K. et al. Altered cytokine export and apoptosis in mice deficient in interleukin-1 beta converting enzyme. Science 267, 2000-2003 (1995).

56. Bouwman, L.I., de Zoete, M.R., Bleumink-Pluym, N.M., Flavell, R.A. \& van Putten, J.P. Inflammasome activation by Campylobacter jejuni. J. Immunol. 193, 4548-4557 (2014). 